\title{
The Postwar Explosion in Radio Astronomy: The US Falls Behind
}

During the Second World War, a number of radar scientists independently discovered powerful radio emission from the Sun. Following the cessation of hostilities, and making use of their wartime experience, scientists, mostly at Jodrell Bank and Cambridge in the UK and in Sydney, Australia, used discarded radar systems to further investigate the complex solar radio emission, discovered powerful radio emission from old supernova explosions, and even more powerful radio sources from what later became known as radio galaxies. Encouraged by their early successes with relatively primitive equipment and the potential for new discoveries, scientists in the UK, Australia, the USSR, and the Netherlands developed plans to build more powerful radio telescopes and sophisticated new instrumentation.

In the US, support for radio astronomy was largely driven by Cold War defense concerns, with funding mostly from the Office of Naval Research (ONR) and the Air Force Office of Scientific Research (AFOSR). Radio astronomy projects were begun at several universities, research institutes, and government and military laboratories. Despite the considerable investment in resources under the direction of skilled scientists and engineers, the early American radio astronomy research did not have the same impact as the programs in the UK and Australia.

In this chapter we discuss the postwar explosion in radio astronomy leading to concerns that the United States had fallen behind other countries, namely the UK and Australia, in this rapidly developing field of astronomy with obvious commercial and military implications to the growing Cold War environment. These concerns, whether real, imagined, or invented, would lead to efforts, begun in the mid-1950s, to establish a national radio astronomy facility to compete with the rapidly developing programs in Europe and Australia. Edge (1984), Edge and Mulkay (1976), Elbers (2017), Frater et al. (2017), Kellermann (2012), Orchiston et al. (2007), Robertson (1992), and especially Sullivan (2009) have described these developments in more detail, while more 
personal accounts are given by Bolton (1982), Bowen (1984, 1987), Bracewell (2005), Christiansen (1984), Denisse (1984), Ginzburg (1984), GrahamSmith (2005), Haddock (1984), Hanbury Brown (1991), Hey (1973), Kraus (1984, 1995), Lovell (1984a, b, 1990), Mills (1984, 2006), Salomonovich (1984), Tanaka (1984), Wang (2009), and in the compilations by Kellermann and Sheets (1984) and Sullivan (1984).

\subsection{Postwar Radio Astronomy}

By the end of WWII, it was increasingly apparent that the discoveries by Jansky and Reber, as well as the wartime serendipitous discoveries of powerful radio emission from the Sun, presented a new way to study the Universe. Scientists and engineers in a number of countries, particularly in Australia, the UK, The Netherlands, Russia, and in the US turned their attention to following up these opportunities. With a few exceptions, namely in the Netherlands and at Harvard University and Berkeley, these programs were initiated by scientists and engineers with backgrounds in physics and radio science, rather than by astronomers.

The MIT Radiation Laboratory The MIT Radiation Laboratory, known as the "Rad Lab," was a remarkable example of wartime success that employed over 3500 skilled scientists and spent nearly $\$ 4$ million a month developing and producing radar systems. As the end of WWII approached, Robert (Bob) Dicke, then a young PhD from the University of Rochester, together with other Rad Lab colleagues, conducted a series of groundbreaking experiments that would later have a major impact on the development of radio astronomy. Using a small 1.5 foot $(46 \mathrm{~cm})$ dish, Dicke and Berringer (1946) measured the $1.25 \mathrm{~cm}(24 \mathrm{GHz})$ radio emission from the Sun and the Moon, and reported black body temperatures of $11,000 \mathrm{~K}$ and of $292 \mathrm{~K}$ respectively. During a partial solar eclipse on 9 July 1945, they demonstrated that the $1.25 \mathrm{~cm}$ emission from the Sun came from the same region as the visual solar disk. These measurements were at by far the shortest wavelength that had been used for radio astronomy. During the same period, Dicke et al. (1946) observed, for the first time, the thermal radio emission from the atmosphere at $1.0 \mathrm{~cm}, 1.25 \mathrm{~cm}$, and $1.5 \mathrm{~cm}$, confirming that the suspected opacity that had been indicated by erratic $1.3 \mathrm{~cm}$ radar operation was due to atmospheric water vapor. They were unable to detect any radio emission from any stars, and also put an upper limit of 20 degrees on any "radiation from cosmic matter," although Dicke did not appreciate its importance until two decades later.

In order to suppress the effect of receiver gain instabilities which could mimic the response of a cosmic source, in all of these studies, Dicke (1946) rapidly switched his receiver between the antenna and a reference source 30 times per second, and measured only the difference signal which was nearly independent of receiver gain fluctuations. This synchronous detection scheme which has come to be known a "Dicke switch" became the basis of all non- 
interferometric radio astronomy continuum measurements and, in a modified form, spectroscopic observations as well.

This series of observations, which took place during the summer of 1945, led to seven key results: the first radio astronomy observations at short centimeter wavelengths, the first detection of radio emission from the Moon, the first measurements of microwave attenuation by atmospheric water vapor, the development of the Dicke switch, the quantitative development of the fundamental radiometer equation, calibration using a variable temperature resistive load, and the first upper limit on any isotropic cosmic background radiation. While these contributions of Dicke and his colleagues marked the beginning of the postwar era of radio astronomy, at the time they went largely unnoticed by the astronomical community.

Radio Astronomy in the UK Following the end of WWII, radio astronomy in Britain was pursued in three places: at Cambridge University under Martin Ryle, at the University of Manchester's Jodrell Bank Observatory under Bernard Lovell, and at the UK Army Operational Research Group (AORG) under J. Stanley Hey. Ryle, Lovell, and Hey had each been involved in wartime radar research. Only Ryle and Lovell returned to university life after the War, while Hey began a radio astronomy program within the AORG.

Hey's background was in physics, having received a Masters' degree in X-ray crystallography at Manchester University (Hey 1973). He had no previous training in radio engineering prior to a six week military training program, and no background in astronomy. After seven years leading the AORG radio astronomy program at Richmond Park, just 10 miles from downtown London, Hey built a research group at Malvern where he later constructed a twoelement variable spacing radio interferometer with baselines up to $1 \mathrm{~km}$.

Lovell returned to the University of Manchester to set up a radar system first to study cosmic rays and then to study the ionization trails left by meteors. To enhance their sensitivity, Lovell and J.A. Clegg built a fixed 218 foot $(66 \mathrm{~m})$ reflector at Jodrell Bank near Manchester. Although Lovell planned to use the telescope for a meteor scatter program, he was joined by Robert Hanbury Brown and Cyril Hazard, who used the dish for a variety of radio astronomy programs at 1.89 meters $(159 \mathrm{MHz}$ ), including observations of M31 (Hanbury Brown and Hazard 1951) and a survey that disclosed 23 discrete radio sources (Hanbury Brown and Hazard 1953). Inspired by the success of the 218 foot dish, Lovell went on to build the iconic fully steerable 250 foot (76 meter) dish, which went into operation in 1957 (Sect. 6.6) following an agonizing engineering and financial near-fiasco that almost sent Lovell to prison (Lovell 1984b, 1990). Later, under the leadership of Henry Palmer, Jodrell Bank radio astronomers also built a series of radio-linked interferometers of ever increasing angular resolution (Sect. 8.1).

Radio astronomy at Cambridge was established under the forceful leadership of Martin Ryle, who, over a period of several decades, guided the development of interferometer systems of ever increasing sophistication and angular 
resolution, coupled with the formulation of the concepts of aperture synthesis (Sect. 7.1). With these innovative radio telescopes, Ryle and a group of talented students made a series of radio source surveys, including the IC (Ryle et al. 1950), 2C (Shakeshaft et al. 1955), 3C (Edge et al. 1959), and its revision 3CR (Bennett 1962). The 3CR survey, in particular, became for many years the basis of detailed radio source studies, at least in the Northern Hemisphere.

The Australian CSIRO Radiophysics Laboratory As the War was winding down, E.G. (Taffy) Bowen left the MIT Radiation Lab to take up an appointment at the Australian Commonwealth Scientific and Industrial Research Organization (CSIRO, then called CSIR or Council for Scientific and Industrial Research). Two years later he became Chief of the CSIRO Radiophysics Laboratory, where he began programs in cloud physics and radio astronomy. Joseph L. Pawsey, who had joined the Radiophysics Lab in 1940, led the new radio astronomy program. John Bolton (Kellermann 1996; Robertson 2017), Paul Wild (Frater and Ekers 2012), Bernard (Bernie) Mills (Frater et al. 2017, p. 23), and Wilbur Norman (Chris) Christiansen (Frater et al. 2017, p. 59) all joined the group, and along with Pawsey, began their own independent research programs.

Pawsey (1946), with support from Bolton and Gordon Stanley, observed the Sun from field stations at Collaroy and Dover Heights in the Sydney suburbs, confirming that the intense radio emission from the Sun was associated with sunspot activity. Although their radar antenna did not have sufficient angular resolution to determine the location of the radio emission on the Sun, Pawsey used a trick to enhance the resolution of his simple antenna.

During WWII, shipboard radar operators would sometimes note that when an aircraft was approaching at low elevation, two signals were received, one directly from the aircraft and one reflected off the ocean. The two signals interfered with each other, and as the angle of approach changed due to the changing differential path length, there would be a series of interference maxima and minima. ${ }^{1}$ Pawsey exploited this "sea interferometer" effect to study the structure of solar radio emission using a small antenna located on a Dover Heights cliff overlooking the Pacific Ocean (McCready et al. 1947). ${ }^{2}$

Both Christiansen and Wild continued to study solar radio emission. Initially, Wild concentrated on studying the solar dynamic spectra, and established the now standard nomenclature used to classify solar radio bursts. He later built a 96-element circular array to obtain dynamic images of the Sun with a resolution of up to about 2 arcmin (Wild 1967), and went on to become first Chief of the CSIRO Division of Radiophysics and then Chairman of CSIRO. Christiansen built a series of interferometer arrays of increasing sophistication, and developed novel synthesis imaging techniques complementing the development of aperture synthesis by the Cambridge group (Sect. 7.1). Bernie Mills developed an alternate approach to obtain high resolution by building large cross arrays (known as a Mills Cross) with each arm built of many simple dipoles (Mills and Little 1953). John Bolton, assisted by Gordon 
Stanley and Bruce Slee, identified the first radio sources with optical counterparts (Sect. 2.3). Together with Stanley, Bolton left Australia in 1955 to start the radio astronomy program at Caltech, only to return six years later to direct the operation of the Parkes 210 foot radio telescope (Kellermann 1996; Robertson 2017). However, when Bowen decided to concentrate the Radiophysics resources on the new Parkes 210 foot radio telescope, Mills and Christiansen left CSIRO to join the schools of physics and electrical engineering at the University of Sydney (Robertson 1992; Frater et al. 2017).

Radio Astronomy in Europe Although recovery from the devastating effects of WWII was slow, modest radio astronomy programs began in the Netherlands and in France using captured German radar systems, particularly the 7.5 meter Würzburg parabolic dishes.

The French radio astronomy program began under the leadership of JeanFrançois Denisse and Jean-Louis Steinberg, who were later joined by Émile Blum and others. As France was occupied from the early days of WWII, French scientists were somewhat isolated from the technical advances that led to the rapid development of radio astronomy in the "Anglo-Saxon world" (Denisse 1984). As elsewhere, the first programs were in solar radio astronomy, largely because the Sun was the brightest source in the sky and did not require sophisticated equipment. Other observations included a $900 \mathrm{MHz}$ survey of the galactic plane using a 7.5 meter Würzburg antenna (Denisse et al. 1955). Later Lequeux et al. (1959) built a variable spacing interferometer at the Nançay Observatory using two Würzburg antennas with baselines up to $1.5 \mathrm{~km}$, which was used by Lequeux (1962) at $1.4 \mathrm{GHz}$ to observe the structure of 40 discrete sources, many for the first time. The Nançay interferometer observations were among the first to demonstrate the double nature of many extragalactic radio sources. However, with limited sensitivity and lack of phase stability, the Nançay interferometer could not compete with the almost contemporaneous Caltech Owens Valley Interferometer.

Unlike those in other countries, the Dutch radio astronomy program began not with radio physicists, but under the leadership of the well-known astronomer, Jan Oort. Indeed, due to the lack of scientists experienced in radio instrumentation, the Dutch program initially suffered until the electronics engineer C. Alexander (Lex) Muller was recruited from Dutch industry by Oort. As in other countries, the Dutch radio astronomy program was initially focused on the Sun. But for the decade after the 1951 detection of the hydrogen $21 \mathrm{~cm}$ line (Sect. 2.4) it was nearly exclusively devoted to $21 \mathrm{~cm}$ research, with the goal of using Doppler shifts to determine the kinematics of the Galaxy.

Radio astronomy in Sweden started in 1949 when Olof Rydbeck brought five Würzburgs from the remote Norwegian coast to his Onsala Space Observatory (OSO) which was part of the Chalmers University of Technology. Early research was concentrated on the Sun and $21 \mathrm{~cm}$ observations of galactic structure. Following the construction of 25 and 20 meter telescopes, the OSO became one of the European leaders in very long baseline interferometry 
(VLBI) (Sect. 8.1) and molecular spectroscopy. Some of the first successful maser amplifiers used for radio astronomy were developed at the OSO. Hein Hvatum, later to become head of NRAO technical programs, began his career at Chalmers under the strong supervision of Rydbeck. Like many of the other pioneers of radio astronomy, Rydbeck's background was in electronics and radiophysics (Radhakrishnan 2006). Although Sweden was neutral during WWII, Rydbeck was one of the leaders in developing the wartime radar defenses needed to maintain Sweden's neutrality.

In Germany, research in radio physics was initially forbidden by the occupying Allied powers but developed starting in the 1950s, especially after 1962 when Otto Hachenberg became director of the University of Bonn's 25 meter Stockert radio telescope (Sect. 9.2).

Radio Astronomy in Japan As related by Tanaka (1984), just as in Germany, the start of radio astronomy in Japan was delayed by their defeat in WWII. Separate projects using relatively simple equipment to study the Sun were started by Minoru Oda, T. Hatanaka, A. Kimpara, Koichi Shimoda, and Haruo Tanaka. Later Tanaka led the development of a five-element interferometer for high resolution solar imaging which was expanded to eight elements in 1954. As pointed out by Ishiguro et al. (2012), perhaps one of the most important Japanese contributions to early radio astronomy was the development by Shintaro Uda and his professor Hidetsugu Yagi of the Yagi-Uda antenna, more commonly known as the Yagi antenna, which was used world-wide in many of the early radio astronomy systems, as well as in a multitude of other short wave communication systems. With the creation of the Nobeyama Radio Observatory in 1970 following the construction of the 84-element heliograph, the six-element millimeter array of precision 10 meter dishes, the 45 meter radio telescope (Sect. 10.5), and the establishment of a vigorous space VLBI (Sect. 8.9) program, Japan became a major player in cosmic as well as in solar radio astronomy, and a pioneer in the development of millimeter wave interferometry (Sect. 10.5).

Radio Astronomy Behind the Iron Curtain As in Western countries, early radio astronomy research in the USSR was primarily the domain of scientists proficient in radio physics and electronics who had developed their skills while working on wartime radar programs. Although postwar support for radio astronomy in essentially all countries had its origin in military radar, in most countries it was implemented in universities and in civilian research laboratories. However, in the USSR radio astronomy remained within military-oriented and tightly controlled laboratories that limited the distribution of research findings. Even when results of radio astronomy investigations were published in the open literature, critical data on the instrumentation and techniques used in the observations were often restricted, leading to skepticism or disregard of Soviet papers by Western scientists. Within the Soviet Union itself, there was little communication among the different radio astronomy groups who were 
competing for limited resources, and even less contact with the broader international astronomical community.

Starting in 1958, many of the most important Soviet journals were translated into English, but Soviet observational results had little impact outside of the USSR. In contrast, however, the theoretical work of people like Iosef Shklovsky, Solomon Pikel'ner, Vitaly Ginzburg, and Yakov Zel'dovich in Moscow, as well as Viktor Ambartsumian in Armenia, and later their students, Nikolai Kardashev, Igor Novikov, Vyacheslav Slysh, and Rashid Sunyaev, was widely recognized and greatly influenced both theoretical thinking as well as motivating new observational programs in the US, Europe, and Australia. Indeed, the English language translation of Shklovsky's book, Cosmic Radio Waves (Shklovsky 1960) was used by generations of students around the world.

Postwar Soviet observational radio astronomy programs were led by S.E. Khaikin (Lebedev Physical Institute), N.D. Papalesky (Lebedev), V.S. Troitsky (Gorky), and V.V. Vitkevich (Lebedev). One of the earliest programs involved a 1947 eclipse expedition to Brazil. Using a 1.5 meter $(200 \mathrm{MHz})$ phased dipole array mounted on the deck of their ship, Khaikin had the ship's captain maneuver the vessel to track the Sun during the solar eclipse and was able to demonstrate that the radio emission came from the much larger coronal region rather than the totally eclipsed Sun (Salomonovich 1984). Starting in 1948, under the leadership of Vitkevitch, Lebedev radio astronomers set up a series of observing stations on the south coast of Crimea, including a refurbished captured German Würzburg-Riese antenna. In 1959, Lebedev constructed a 22 meter (72 foot) precision dish at one of the sites on the shore of the Black Sea that operated at wavelengths as short as $8 \mathrm{~mm}$, and for a long time was the largest radio telescope in the world operating at such short wavelengths. Twenty years later, it became the focal point of the first US-USSR VLBI observations (Sect. 8.2).

Radio Astronomy in China As in other countries, early post-war radio astronomy in China concentrated on solar research at meter wavelengths, but with instrumentation imported from the USSR. Even more than in the USSR, radio astronomy activities in China were cloaked in secrecy and hidden from Western scientists. Likewise Chinese scientists had little contact with the West (Wang 2009). But in remarkable contrast to pervading policies, starting in 1963, Chris Christiansen from Australia made more than a dozen visits to China, where he helped implement a series of advanced radio astronomy arrays. As a result of his frequent trips to China, Christiansen for many years was denied visas to visit the United States. Following the end in 1976 of the Cultural Revolution in China and the resumption of diplomatic relations with Western countries, exchanges between Chinese and Western scientists flourished; a new generation of young radio astronomers were trained, and China went on to develop strong programs in radio astronomy. 


\subsection{Radio Waves From the Sun ${ }^{3}$}

Although the Sun is the strongest radio source in the sky other than the Milky Way Galaxy, especially during periods of solar activity, neither Jansky nor Reber, until 1944, were able to detect radio emission from the Sun. However, during the 1920s and 1930s US, British, and Japanese amateur radio operators, mostly operating in the $10 \mathrm{~m}(28 \mathrm{MHz})$ band, reported noise or hissing that was probably due to radio emission from the Sun during periods of intense solar activity. Probably the first documented report of solar radio emission was by the British radio amateur D.W. Heightman who operated the amateur radio station G6DH. Heightman (1936) reported a "smooth hissing sound when listened to on a receiver," which he suggested, "apparently originates on the Sun, since it has only been heard during daylight."

Solar radio emission was independently detected and documented on multiple occasions during World War II by radar operators in the UK, in Germany (Schott 1947), in Japan (Tanaka 1984), and in the South Pacific by Elizabeth Alexander. ${ }^{4}$ Hey (1973, p. 15) and Lovell (1984a) have described one of the best known and perhaps the most dramatic serendipitous detection of solar radio emission which occurred in February 1942 when Britain was on high alert for an impending German invasion. On 12 February two German warships, the Scharnhorst and the Gneisenau, passed through the English Channel undetected by the jammed $4.2 \mathrm{~m}(71.4 \mathrm{MHz})$ British coastal radar. Concerned about the effectiveness of their coastal defense radar chain, the UK War Office assigned the AORG's J.S. Hey the job of understanding and eliminating the jamming. On 27 and 28 February, the coastal radar systems again became inoperable due to apparent German anti-radar activity, but Hey noticed that the apparent jamming occurred only in the daytime when the radar antennas were pointed toward the Sun, and that it was a time of exceptional sunspot activity. He concluded that the Sun was a source of powerful $4-8 \mathrm{~m}$ (37-75 MHz) radio emission about 100,000 times greater than expected from a $6000 \mathrm{~K}$ black body. Hey described his discovery in a secret report ${ }^{5}$ which became known to scientists in other Allied countries. However, wartime secrecy precluded any publication of his discovery in the scientific literature until the close of hostilities (Hey 1946).

Just a few months later, on 29 June 1942, Bell Labs scientists, A.P. King and George Southworth succeeded in detecting the thermal radio emission from the quiet Sun, first at $9.4 \mathrm{GHz}(3.2 \mathrm{~cm})$ and later at $3.06 \mathrm{GHz}(9.8 \mathrm{~cm})$. As with Hey's discovery, Southworth's study of the radio emission from the quiet Sun wasn't published until after the War (Southworth 1945, 1956). However, Southworth's 1942 classified report was distributed to various groups, including the Harvard College Observatory and the British War Office for further distribution within the British Commonwealth. ${ }^{6}$ During this period, many Bell Labs visitors, including Bowen and Pawsey from Australia, became aware of Southworth's detection of solar radio emission. 
In his $160 \mathrm{MHz}(1.9 \mathrm{~m})$ survey paper, Reber (1944) commented, only in passing, that, in spite of daytime interference, he was able to detect radio emission from the Sun, and realized that the radio emission was more than 100 times more intense than expected from a $6000 \mathrm{~K}$ black body. ${ }^{7}$ Since the earlier observations of solar radio emission by Hey and Southworth remained under military classification until after the end of WWII, Reber's (1944) paper was the first published report of solar radio emission. With great perception, he noted that even if all the stars in the Galaxy radiated with the same intensity as the Sun, that would fail to account for the observed radio emission from the Milky Way. Again, in his later paper on his $480 \mathrm{MHz}(62 \mathrm{~cm})$ survey, Reber (1944) comments only in the last sentence of the paper that he also obtained data on solar radio emission. Although there had been numerous classified observations of solar radio emission in the UK, in the South Pacific, and in the US, Grote Reber (1944), unrestricted by wartime security constraints, was the first to report radio emission from the Sun in the scientific literature and the first to recognize and report the observation of the near 1 million degree solar corona.

While demonstrating his equipment to National Bureau of Standards (NBS) visitors on 21 November 1946, Reber was surprised to observe that intense radio bursts from the Sun drove his chart recorder off scale. ${ }^{8}$ Re-inspection of his earlier records indicated that a similar, but much weaker phenomenon had also occurred on 17 October. In surprising contrast to his earlier slow meticulous work and his 1944 indifference to solar radio emission, Reber dashed off a hastily written letter to Nature (Reber 1946). ${ }^{9}$ In this report, Reber also noted that "the apparent solar temperature [of the quiet Sun] was about a million degrees," in good agreement with the Australian work (Pawsey 1946).

\subsection{Radio Stars and Radio Galaxies}

Grote Reber's $160 \mathrm{MHz}(1.9 \mathrm{~m})$ and especially his $480 \mathrm{MHz}(62 \mathrm{~cm})$ maps indicated several peaks in the radio emission in addition to the one near the Galactic Center, but it would be another serendipitous discovery by J. Stanley Hey, that suggested the first evidence for discrete sources of cosmic radio emission. Hey first learned about Jansky's work from his supervisor when he reported his 1942 discovery of solar jamming of the British coastal radar systems, and had since read Jansky's and Reber's papers in Proc. IRE. Just a few months after the end of the War in Europe, Hey, Phillips, and Parsons (1946b) used a $64 \mathrm{MHz}(5 \mathrm{~m})$ radar antenna to map out the galactic radio emission between declinations -3 and +60 degrees. With a beamwidth of 6 by 15 degrees, they observed the peaks previously noted by Reber at the position of the Galactic Center and also in the Cygnus region near right ascension $20^{\mathrm{hr}} 30^{\mathrm{m}}$ and declination +35 degrees. Continued observations with improved equipment led to a remarkable discovery. Hey, Parsons, and Phillips (1946a) found that the observed emission from the Cygnus region appeared to fluctuate by about $15 \%$ on time scales considerably less than one minute and concluded 
that, "such marked variations could only originate from a small number of discrete sources."

Halfway around the world, in Sydney, Australia, Hey's discovery excited John Bolton. Assisted by Gordon Stanley, Bolton constructed an antenna at Dover Heights overlooking the Pacific Ocean which they used as a $100 \mathrm{MHz}$ sea interferometer to show that the Cygnus source was less than 8 arcmin in extent (Bolton and Stanley 1948). Using this same sea interferometer, Bolton (1948) went on to discover six new discrete sources. Simultaneous observations made from Australia and New Zealand, as well as from sites at Cambridge and Jodrell Bank showed that the intensity variations were independent at the two sites, confirming that they were not intrinsic to the source, but were the result of the signal propagation through the Earth's ionosphere. ${ }^{10}$ After months of painstaking observations, Bolton, Stanley, and Slee (1949) succeeded in measuring the positions of three strong radio sources with accuracy better than half a degree. For the first time it was possible to associate radio sources with known optical objects. They identified the strong radio sources, Taurus A, Centaurus A, and Virgo A with the Crab Nebula, and the galaxies NGC 5128 and M87 respectively. NGC 5128, with its conspicuous dark lane, and M87, with its prominent jet, were well known to astronomers as peculiar galaxies. In their Nature paper, Bolton et al. mostly discussed the nature of the Crab Nebula, but in a few paragraphs near the end of their paper, they commented,

NGC 5128 and NGC 4486 (M87) have not been resolved into stars, so there is little direct evidence that they are true galaxies. If the identification of the radio sources are [sic] accepted, it would indicate that they are [within our own Galaxy].

As implied by the title, of their paper, "Positions of Three Discrete Sources of Galactic Radio-Frequency Radiation," Bolton et al. incorrectly dismissed the extragalactic nature of both Centaurus A and M87. When asked many years later by one of the authors (KIK) why he did not recognize that he had discovered the first radio galaxies, Bolton responded that he knew they were extragalactic, but that he also realized that the corresponding radio luminosity would be orders of magnitude greater than that of our Galaxy and that he was concerned that, in view of their apparent extraordinary luminosity, a conservative Nature referee might hold up publication of the paper. ${ }^{11}$ Nevertheless, in spite of their stated reservation, their 1949 paper is generally regarded as the beginning of extragalactic radio astronomy (Bolton 1982). Yet, for the next few years the nature of discrete radio sources remained controversial within the radio astronomy community. While Ryle and his group at the Cavendish Laboratory continued to refer to radio stars, the extragalactic nature of NGC 5128 and M87 and their powerful radio luminosity was broadly recognized and caught the attention not only of astronomers, but of the physics community as well. How could there be such strong radio sources? Where did the energy come from? How was this energy converted into radio emission? 
The nature of the broader population of radio stars remained uncertain. Among the four brightest sources, there was one galactic supernova remnant, two nearby galaxies, and one unidentified source with no apparent optical counterpart. Mills and Thomas (1951), using a two-element interferometer, succeeded in measuring a more accurate position of the Cygnus A radio source. Mills communicated an apparent identification with a faint galaxy to Rudolph Minkowski, a well-known astronomer at Caltech's Mt. Wilson and Palomar Observatories. But Minkowski could not accept that such a faint, and presumably distant galaxy could be such a strong radio source, and dismissed Mills' identification. Only after F. Graham-Smith (1951) used his two-element interferometer in Cambridge to obtain an even more accurate radio position did Minkowski accept the identification with what appeared to be two galaxies in collision. However, the classic paper by Baade and Minkowski (1954) which reported the identification of Cygnus A gave only footnote recognition to Mills' previous identification.

The identification of the second strongest radio source in the sky with a faint distant galaxy was remarkable. Already both Cambridge and Australian radio astronomers were cataloguing radio sources as much as a 100 times fainter than Cygnus A. The galaxy identified with Cygnus A was near the limit of what was then the largest optical telescope in the word, the 200 inch Palomar telescope. Radio telescopes were apparently poised to open a new approach to exploring the distant and correspondingly early Universe.

The Source Count Controversy After years of considering that the radio stars were part of a galactic population, Martin Ryle was finally convinced, by the observed isotropy of the source distribution, that they were powerful extragalactic objects that could be used to address broad cosmological problems. During the 1950s, there were two fundamentally different approaches to cosmology. On the one hand, Herman Bondi, Thomas Gold, and Fred Hoyle were promoting their non-evolving Steady-State cosmology in which the Universe is, and always was, everywhere the same. A unique characteristic of the Steady-State model was that to maintain the requirement of an unchanging but expanding Universe, their model required the controversial continuous creation of new matter by an unknown process. This stood in contrast to what Hoyle sarcastically called the "big bang" cosmology which has a characteristic time scale beginning with a mysterious singularity and a Universe that evolves with time.

Although even the simplest radio telescopes were able to record data from very great distances, there was a serious problem in using radio measurements for cosmological investigations, since radio observations alone give no indication of distance. Any one radio source might be a nearby galactic supernova, a relatively close low luminosity radio galaxy, or a much more powerful distant radio galaxy. Without optical identifications of radio sources and measurement of their redshift (distance), radio astronomers were unable to determine the distance or even the nature of their radio sources, and the positions of only a 
few of the strongest radio sources were known with sufficient accuracy to identify an optical counterpart. Although most of the fainter radio sources had no optical identification, Martin Ryle and Peter Scheuer at Cambridge reached a profound conclusion from the count of the observed number of radio sources as a function of limiting flux density.

In a static, uniformly filled universe with Euclidian geometry, the number of radio sources, $N$, above a given flux density, $S$, should increase as $S^{-1.5}$ or log $N=-1.5 \log S .{ }^{12}$ Based on the Cambridge $81 \mathrm{MHz} 2 \mathrm{C}$ survey of 1936 sources, of which 1906 sources were small diameter and isotropically distributed, Ryle and Scheuer (1955) concluded that since the observed exponent was closer to -3 than -1.5 , "Attempts to explain the observations in terms of a steady-state theory have little hope of success." In other words, the Cambridge survey contained many more faint, presumably more distant, radio sources than would be expected in a universe with a uniformly distributed population. This meant that in the past, there were either more radio sources or that they were more luminous than at present, concepts referred to as density evolution or luminosity evolution respectively.

However, contemporaneous observations, from the Sydney group led by Bernie Mills using his Mills Cross at essentially the same frequency, disagreed. The Sydney source count had a slope of only -1.7 which they argued, in view of experimental errors, did not differ significantly from -1.5 (Mills and Slee 1957). Moreover a one-to-one comparison of the overlapping survey region, indicated very little agreement, and Mills and Slee concluded that the "discrepancies ... reflect errors in the Cambridge catalogue, and accordingly deductions of cosmological interest derived from its analysis are without foundation." 13 In the ensuing years, Martin Ryle had to deal with challenges from two directions: first from the theoretical side from his Cambridge colleague, Fred Hoyle, who questioned his interpretation of the data, and second, probably more seriously, from the Australian radio astronomers who questioned his data. In several well-publicized lectures Ryle (1955, 1958) vigorously defended his position that the source-count was inconsistent with Steady-State cosmology. Further observations indeed showed that probably three-fourths of the Cambridge 2C sources were not real, but were blends of weaker sources, known as confusion.

A new more reliable Cambridge 3C survey (Edge et al. 1959) of 242 sources away from the galactic plane, made with higher angular resolution, reduced the claimed source count slope to -2.0 . However, the complete Sydney survey of 1658 sources indicated a slope of -1.5 after correction for the effects of noise and confusion, and concluded that "the source counts indicate no divergence from uniformity and no obvious cosmological effects" (Mills et al. 1960). However, Ryle and Clarke (1961) still maintained that "the results appear to provide conclusive evidence against the Steady-State model." The new and more reliable Cambridge 4C and 5C surveys, as well as the Parkes $408 \mathrm{MHz}$ survey, each indicated a slope near -1.8 , consistent with the Sydney value, but still apparently in excess of the steady-state theory (Hewish 1961; Ryle 1968). 
Mills and Hoyle continued to argue that the source count could equally well be interpreted as a local deficiency of only a few dozen strong sources rather than a cosmic excess of weak sources. Ryle responded that even these strong sources are so distant that their distribution is cosmologically significant. ${ }^{14}$ The debate, which went on for more than a decade, was intense, bitter, and personal (Kragh 1996, p. 305), and was only decided in 1965, not from radio source counts, but from the discovery by Penzias and Wilson (1965) of the cosmic microwave background, which was almost universally accepted as convincing evidence for an evolving or Big Bang universe. Ryle was right; we live in an evolutionary universe. However, his arguments were wrong and based on unreliable data. Mills had much better data, consistent with contemporary source counts, but he got the wrong answer.

It is interesting to look back on the source count controversy in light of current data and understanding. Both the Sydney and, especially, the Cambridge data contained serious instrumental errors as well as errors of interpretation. Because there are more weak radio sources than strong sources, even random errors due to noise or confusion make more weak sources appear stronger and strong sources appear weaker at any given flux density level, thus making the observed source count appear steeper than the real value. Secondly, both Ryle and Mills continued to use cumulative counts, where each point was the sum of all the stronger sources. So the data points were not independent and the estimated errors were unrealistically too small. Moreover, features in the source count at some flux density, $S$, would propagate to lower flux densities. ${ }^{15}$ Finally, in a real expanding universe, whether Steady-State or Big Bang, the effect of the redshift is to make sources weaker than would be the case if their flux density fell off as an inverse square of the distance, so the expected slope, in a nonevolving universe should be smaller than -1.5 . Modern radio source counts go about a million times fainter than the Cambridge or Sydney surveys. Except for the strongest one hundred or so sources, the slope of the radio source count is -1.5 or flatter, so it is not clear that the steeper slope reported by the Cambridge observers has any relevance.

The long controversy over the radio source counts did not help the image of radio astronomy within the broader astronomical community. Among radio astronomers, the $2 \mathrm{C}$ fiasco gave interferometers a bad name, which took years to overcome. Although the evidence for an evolving universe is now widely accepted, it is interesting to remember that the Steady-State model made the specific predication that the rate of expansion must increase with time, as confirmed only in 1998 with the Nobel Prize winning discovery that the expansion of the Universe is indeed accelerating and the recognition that $70 \%$ of the Universe is composed of so-called dark energy (Riess et al. 1998; Perlmutter et al. 1999). ${ }^{16}$ It is interesting, however, to speculate how the history of cosmology might have been different if the acceleration of the Universe had been discovered before the discovery of the cosmic microwave background by Penzias and Wilson (1965). 


\subsection{The 21 cm Hydrogen Line: The Beginning of Radio Spectroscopy}

When Jan Oort in the Netherlands learned about Grote Reber's (1944) paper, he suggested to his student, Henk van de Hulst, that he might consider the possibility of spectral line radiation from interstellar hydrogen, the most abundant element in the Universe. ${ }^{17}$ Van de Hulst's (1945) classic paper, written in 1944 and published after the end of the war, primarily dealt with thermal emission from stars and interstellar dust (called "smoke" by van de Hulst), free-free (Bremsstrahlung) emission from ionized hydrogen, neutral hydrogen recombination lines from $\Delta n=1$ atomic transitions, and in only two paragraphs, he discussed the $21 \mathrm{~cm}(1420 \mathrm{MHz})$ hyperfine line from the spin flip transition in neutral hydrogen atoms. ${ }^{18}$ Due to a calculation error (Sullivan 1982, p. 299), Van de Hulst (1945) pessimistically concluded that the recombination lines "are unobservable" due to the Stark effect, and that the hyperfine line "does not appear hopeless," but that "the existence of this line remains speculative."

Shortly after the end of the war, Oort wrote to Reber asking his advice on the construction of an antenna to study interstellar gas. ${ }^{19}$ Keeping his cards close to his chest, Oort made no mention of their plan to search for the $21 \mathrm{~cm}$ hydrogen line. However, about the same time, van de Hulst was visiting the Yerkes Observatory, and went to Wheaton to ask Reber's opinion on whether the $21 \mathrm{~cm}$ line of neutral hydrogen could be detected (Reber 1958). Van de Hulst's inquiry apparently ignited Reber's interest in searching for the hydrogen line himself. He consulted Greenstein ${ }^{20}$ about the probable strength of the $21 \mathrm{~cm}$ line and started to build a $21 \mathrm{~cm}$ receiver and horn feed. He also had ambitious plans to return to $3 \mathrm{~cm}$ and even to work at $1 \mathrm{~cm}$, but unfortunately, he never completed any of these projects, perhaps discouraged by van de Hulst's pessimism about detecting the hydrogen line.

Later, Iosef Shklovsky (1949) published a paper in Russian discussing the possibility of detecting monochromatic radio emission. Shklovsky had not seen the Dutch language paper by van de Hulst, but was aware only of the brief mention by Reber and Greenstein (1947) in their review of radio astronomy. Working independently of van de Hulst, Shklovsky calculated the transition frequency, but reached a more optimistic conclusion, writing,

In summary, we must say that the resources of contemporary radio techniques fully allow one to detect and measure the monochromatic radio emission of the galaxy.... It is of fundamental importance that the interstellar hydrogen can in this case be directly studied in its ground state. In direct contrast, all methods now existing in astrophysics allow one to determine the population of only the excited states of the hydrogen atom.... Study of the intensity distribution of monochromatic radio emission from the Milky Way would give the distribution of interstellar gas in various regions of the galaxy.... Soviet radiophysicists and astronomers should endeavor to solve this absorbing and important problem. 
Shklovsky's paper did not go unrecognized in the US. Following a year teaching astronomy at Amherst and three years as a Second Lieutenant in the US Naval Air Corp, where he took courses in electronics at Princeton and MIT, Harold I. (Doc) Ewen, while maintaining an appointment as a Naval Reserve officer, entered Harvard graduate school to pursue a $\mathrm{PhD}$ in physics. ${ }^{21}$ His commanding officer was Donald Menzel, then a Captain in the Naval Reserves. Menzel sent Ewen for a two week assignment at the National Bureau of Standards, where he worked under the supervision of Grote Reber. Returning to Harvard, Ewen contacted Edward Purcell, who suggested that Ewen investigate the literature on the neutral hydrogen hyperfine line. Using his naval connections, Ewen was able to obtain a translation of van de Hulst's (1945) paper from the Naval Research Laboratory (NRL). Although discouraged by van de Hulst's conclusion, Ewen and Purcell agreed that Ewen's thesis topic would be to assemble a microwave spectrometer with the objective of setting the "level of non-detectability" of galactic hydrogen. Meanwhile, to earn money, Ewen worked nearly full time building a proton accelerator at the Harvard Cyclotron Lab.

Resigned to a negative thesis, Ewen later claimed that he had little interest in the project until he saw Shklovsky's 1949 paper. $^{22}$ Although he had assumed that the Dutch were not going to try to detect the hydrogen line, he then worried that the Soviets, encouraged by Shklovsky's remarks, were probably already hard at work in pursuit of a detection. Full of enthusiasm and excitement, Shklovsky had tried to convince Victor Vitkevich, the leading Soviet radio astronomer, to attempt to detect the $21 \mathrm{~cm}$ line. But, after showing some initial interest, according to Shklovsky (1982), Vitkevich suggested that while it was easy for theoreticians to throw out ideas, experimental radio astronomy is real work and that he had enough ideas of his own. Years later, Shklovsky noted, Vitkevich admitted that he had been intimidated by Lev Landau, the famous Russian physicist, who claimed that the whole idea was "pathological."

Purcell was able to obtain a $\$ 500$ grant from the American Academy of Arts and Science that enabled Ewen to purchase components to build a 21 centimeter receiver and to construct a pyramidal horn antenna (Figs. 2.1 and 2.2). For the next year, while continuing to work trying to produce a proton beam at the Cyclotron Lab in the daytime, on weekends Ewen worked on his hydrogen line system. He built a horn out of plywood and copper foil, which he pointed outside the window of the Lyman Physics Building at a declination of -5 degrees. The instrumentation needed to measure the $21 \mathrm{~cm}$ hydrogen line was developed using test equipment "borrowed" from the Cyclotron Lab on Friday afternoon and returned by Monday morning. At Purcell's suggestion, Ewen designed and built a frequency switched system patterned after Dicke's load switching radiometer. ${ }^{23}$ His search for the hydrogen line was aided by the recent laboratory measurement of the line frequency of $1420.405 \mathrm{MHz}$ (Kusch and Prodell 1950), so he knew precisely where to tune his receiver. Due to Doppler broadening, the galactic hydrogen emission is smoothed over a broad frequency range. With comparable signal strength in both the line and reference 


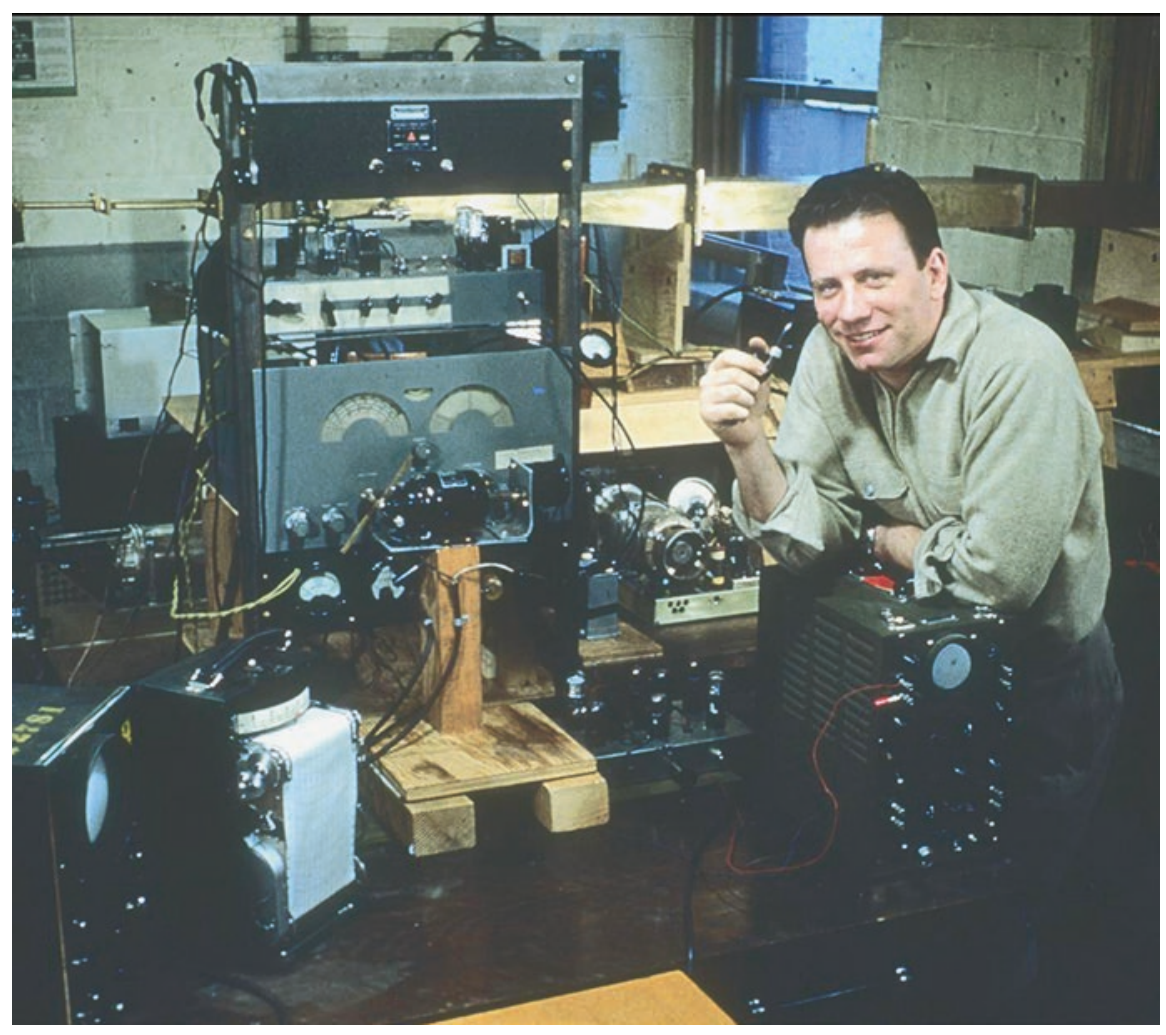

Fig. 2.1 Doc Ewen and his $21 \mathrm{~cm}$ receiver at Harvard University, March 1951. Credit: NRAO/AUI/NSF

channels separated by only $10 \mathrm{kHz}$, Ewen initially missed seeing the $21 \mathrm{~cm}$ hydrogen signal. Supported by another $\$ 300$ from Purcell's personal funds, Ewen purchased a commercial shortwave receiver to use as the final stage of his receiver. This permitted him to use a sufficiently wide $75 \mathrm{kHz}$ separation between the signal and reference frequency to avoid any galactic hydrogen radiation in the reference channel.

On 25 March 1951, Ewen detected the galactic $21 \mathrm{~cm}$ radiation peaking near 18 hours right ascension, although the observed frequency was about $150 \mathrm{kHz}$ above the laboratory value. A quick consultation with the Harvard astronomers confirmed Ewen's suspicion that the discrepancy was due to the Doppler shift caused by the Earth's orbital motion and the motion of the Solar System. At the time, van de Hulst happened to be visiting Harvard, where he was teaching a course in astronomy. There had been no previous contact between Ewen and van de Hulst until, at Purcell's suggestion, Ewen informed him of their successful detection. Ewen recalled that van de Hulst seemed "shocked" and did not appear particularly "delighted to learn that his 'long 


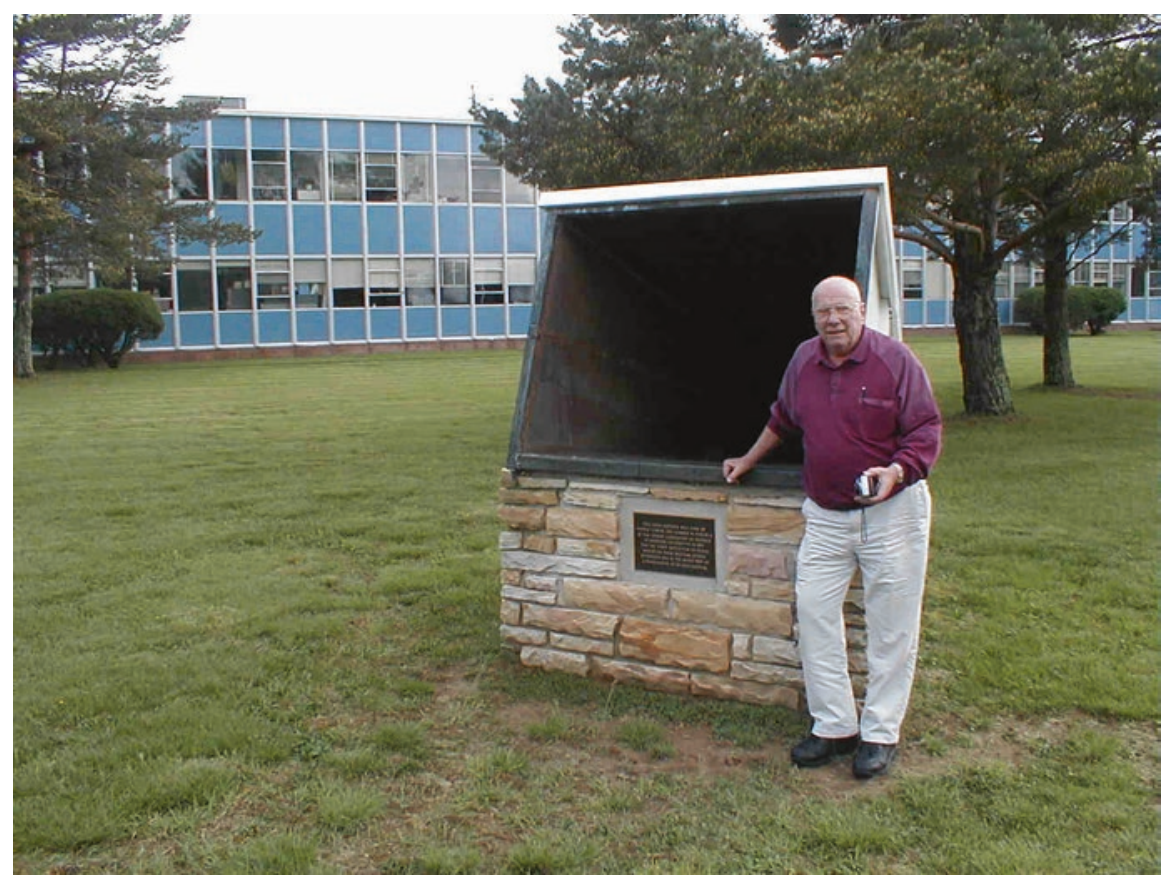

Fig. 2.2 Doc Ewen and his original horn antenna at NRAO in Green Bank, West Virginia, May 2001. Credit: NRAO/AUI/NSF

shot speculation' proved to be correct." ${ }^{24}$ Only then did Ewen learn that the Dutch had been unsuccessfully trying for years to detect the $21 \mathrm{~cm}$ line. Although van de Hulst had not expected that the hydrogen line could be detected, he explained that Oort appreciated its importance, and continued to encourage the attempt at Leiden. Van de Hulst peppered Ewen with questions about where he found hydrogen emission in the Galaxy and how to build the receiver. Lex Muller adopted the Harvard frequency switching concept, and on 11 May 1951, using a 7.5 meter Würzburg antenna at Kootwijk in central Holland, Muller and Oort (1951) were able to confirm the Harvard result. Characteristically, not satisfied with a simple confirmation, the Dutch paper included observations made at different positions in the Galaxy and commented on the distribution of hydrogen gas and the kinematics of the Galaxy.

Another Harvard visitor at this time was Frank Kerr from the CSIRO Radiophysics Laboratory. Following a visit to Ewen's lab in early April 1951, Kerr informed Joe Pawsey in Australia about Ewen's successful detection of galactic hydrogen. Kerr explained Purcell's frequency comparison technique and requested possible confirmation from Australia. Pawsey wrote back to Purcell that they had not previously been working on the hydrogen line detection, but upon hearing from Kerr of Harvard's success, he informed Purcell they had initiated two separate programs to confirm Ewen's result. 
Ewen claimed that he wrote his 47 page thesis in three days and defended it in May, just two months after his detection of the hydrogen line. After managing to finagle his way through his German language exam, ${ }^{25}$ Ewen received his PhD in Physics from Harvard. Ewen and Purcell (1951a) presented their discovery at the Schenectady meeting of the American Physical Society on 16 June 1951. The Harvard (Ewen and Purcell 1951b) and Dutch (Muller and Oort 1951) papers were sent to Nature on June 14 and 20 respectively and published in successive papers in the 1 September 1951 issue. The Dutch paper was followed by a brief note from Joe Pawsey, dated 12 July, announcing that Christiansen and Hindman had also confirmed Ewen's discovery but with no details. Christiansen and Hindman (1952a, b) published a brief report in Observatory followed by a more detailed paper in the Australian Journal of Scientific Research. Using a tracking antenna, they showed that the distribution of galactic hydrogen was concentrated along the galactic plane and based on observed frequency splitting of the line profiles, they suggested evidence for the galactic spiral arms.

All three programs were enabled by using frequency switching, and all three programs used a variable capacitance to scan in frequency. Ewen later commented that there was never a real competition to make the first detection. But rather "we gave them the Heath kit, the parameter values of the signal, and instructions on where to look. The Australians freely admit this, the Dutch never have."26

A few months after these papers appeared, Paul Wild from Australia submitted a paper to the Astrophysical Journal (Wild 1952) discussing expected frequencies for various possible hydrogen atom transitions, including high order recombination lines, fine structure lines, as well as the spin-flip hyperfine structure line and the effect of Zeeman splitting in a magnetic field. Although not submitted until after Wild became aware of the actual detections at Harvard, Leiden, and Sydney, his paper was based on two pre-discovery papers which he wrote in 1949 for private distribution. ${ }^{27}$

When Grote Reber learned about Harvard's successful detection of hydrogen, he wrote to congratulate Ewen and urged him to try for the $92 \mathrm{~cm}$ $(327 \mathrm{MHz})$ deuterium line. ${ }^{28}$ But Ewen had other ideas. He enrolled in the Harvard Business School to pursue an MBA to complement his PhD in Physics. He was called to active duty during the Korean War, but served for only a month before negotiating his return to Cambridge as a civilian. Although he later participated in several radio astronomy conferences, and took part in the search for a site for NRAO, Ewen never continued his pioneering research on the $21 \mathrm{~cm}$ hydrogen line, but helped Bart Bok start the Harvard radio astronomy project and served as co-director with Bok. Together with his friend, Geoff Knight, Ewen started his own business, the Ewen-Knight Company to construct a new hydrogen line receiver for Harvard. Ewen Knight went on to be a major supplier of equipment to many radio observatories including NRAO. Building on Karl Jansky's patent, Ewen later developed a form of radio 
sextant which was used on Polaris submarines when they needed to know their location, independent of weather conditions, before firing their missiles.

\subsection{Early US University Radio Astronomy Programs}

Unlike in other countries, US postwar radio astronomy was largely funded through contracts between universities and the military research programs at the Office of Naval Research (ONR) and the Air Force Office of Scientific Research (AFOSR). Still buoyed by the scientific successes of WWII and further stimulated by the Korean War, in the 1950s US universities, including Caltech, Cornell, Stanford, the University of California, and the University Illinois, received generous support from the ONR and the AFOSR to begin modest radio astronomy programs. Other programs in the US began at the Carnegie Institution's Department of Terrestrial Magnetism (DTM) and at the Naval Research Laboratory (NRL). Starting in 1948, Grote Reber pursued radio astronomy programs at the National Bureau of Standards (NBS) until 1951 and then, with modest support from the New York based Research Corporation, in Hawaii. Except at Harvard and Ohio State, the National Science Foundation played little role in these early years of US radio astronomy.

Harvard University As Ewen later remarked, "The discovery of the $21 \mathrm{~cm}$ interstellar hydrogen line radiation in 1951 initially generated little interest at Harvard." 29 However, after several years of military service, Campbell Wade had just returned to Harvard to complete his undergraduate degree in astronomy. While in the military, Wade had acquired some skills in radio technology. He heard about Ewen's discovery and was excited about the prospects for further work in what he foresaw as an exciting new field of astronomy. $\mathrm{He}$ naturally went to Bart Bok, who was widely recognized among the student body as probably the friendliest Harvard astronomy professor, and who had just returned from an extended period in South Africa. Bok contacted his friend Ed Purcell to discuss the prospects for establishing a radio astronomy program at Harvard, but he had no encouragement from his peers. Walter Baade suggested that at age 51, Bok was too old to get involved in this new field with uncertain prospects. After all, Jansky and Reber had measured the continuous radiation from the Galaxy and Ewen and Purcell had discovered the $21 \mathrm{~cm}$ radiation from galactic hydrogen. Nothing was left! Nevertheless, with Purcell's encouragement, Bok sent Wade, along with two senior graduate students, David Heeschen and Edward Lilley, to see Ewen and learn how to operate his equipment. However, the three of them found the room locked and learned that all of the equipment had been sent to DTM in Washington, where Merle Tuve wanted to start a new radio astronomy program. ${ }^{30}$

A few months later Bok obtained the first NSF grant for radio astronomy, for $\$ 32,000$ to purchase a 24 foot $(7.3$ meter $)$ antenna, but he needed additional funds for the instrumentation. He convinced Mabel Agassiz, a wealthy Harvard benefactor, to contribute another $\$ 40,000$ toward the construction 
and operation of the telescope that was located in the town of Harvard, Massachusetts, about 30 miles distant from the Harvard University campus in Cambridge. Bok was able to get additional support from the University, as well as from the Research Corporation, to operate the telescope and to provide additional instrumentation. Ewen built a new hydrogen line receiver and Heeschen, Lilley, and Wade helped to put the telescope into operation and conducted the first research programs. Heeschen and Lilly went on to be among the first Americans to receive their $\mathrm{PhD}$ degree for work in radio astronomy, when they both passed their Harvard oral exams on 26 November 1954. ${ }^{31}$ They were followed by T.K. (Kochu) Menon in 1956, Thomas Matthews in 1956, W.E. (Bill) Howard III in 1958, May A. Kaftan-Kassim in 1958, Nannielou (Nan) Hepburn Dieter Conklin in 1958, Frank Drake in 1958, and Campbell (Cam) Wade in 1958.

In 1954, Bok and Dave Heeschen convinced Mrs. Agassiz to help fund the design of a 60 foot (18 meter) radio telescope. Only in this way, they explained, could American astronomers keep up with radio astronomers in England, and the Netherlands, who were using their captured German Würzburg antennas for a variety of research programs. Bok received another NSF grant of $\$ 132,000$ for the construction and initial operation of the telescope. When completed in the spring of 1956 by D. S. Kennedy and Co., the Harvard 60 foot dish became one of the largest fully steerable radio telescopes in the world and the largest equatorially mounted telescope. ${ }^{32}$ The Ewen-Knight Corporation built new receiving equipment for $21 \mathrm{~cm}$ research, including a 20 channel spectrometer. NSF Director Alan Waterman spoke to more than 200 people at the dedication of Harvard's new radio telescope, referring to radio astronomy as "a new window on the universe," and acknowledging the responsibility of the Federal Government to support basic research "when such support is necessary and in the interest of science." 33

Unlike the radio astronomy programs in Australia and the UK, the Harvard project was managed by astronomers, not radio scientists. Even at the other American universities involved in radio astronomy the programs were led by people with backgrounds in radiophysics such as William Gordon, Charlie Seeger, and Marshall Cohen at Cornell, John Kraus at Ohio State, and Ronald Bracewell at Stanford. Bok insisted that Harvard students had to learn astronomy as well as radio technology. The research programs at the 24 and 60 foot antennas were heavily concentrated on studying galactic $\mathrm{H} \mathrm{I}$ emission, particularly in the direction of various nebulosities, star clusters, and the Galactic Center along with the delineation of the Galaxy's spiral structure. However, they found time to search, unsuccessfully, for the galactic hydroxyl $(\mathrm{OH}) \mathrm{mol}-$ ecule based on a calculated frequency provided by Charlie Townes (1957), and Heeschen observed H I in the Coma cluster (Fig. 2.3).

Dave Heeschen, Frank Drake, Kochu Menon, Cam Wade, and Bill Howard all went on to become prominent members of the NRAO Scientific Staff, with Heeschen later becoming the NRAO director from 1962 to 1978. Drake became director of the Cornell Arecibo Observatory. Nan Dieter went to the 


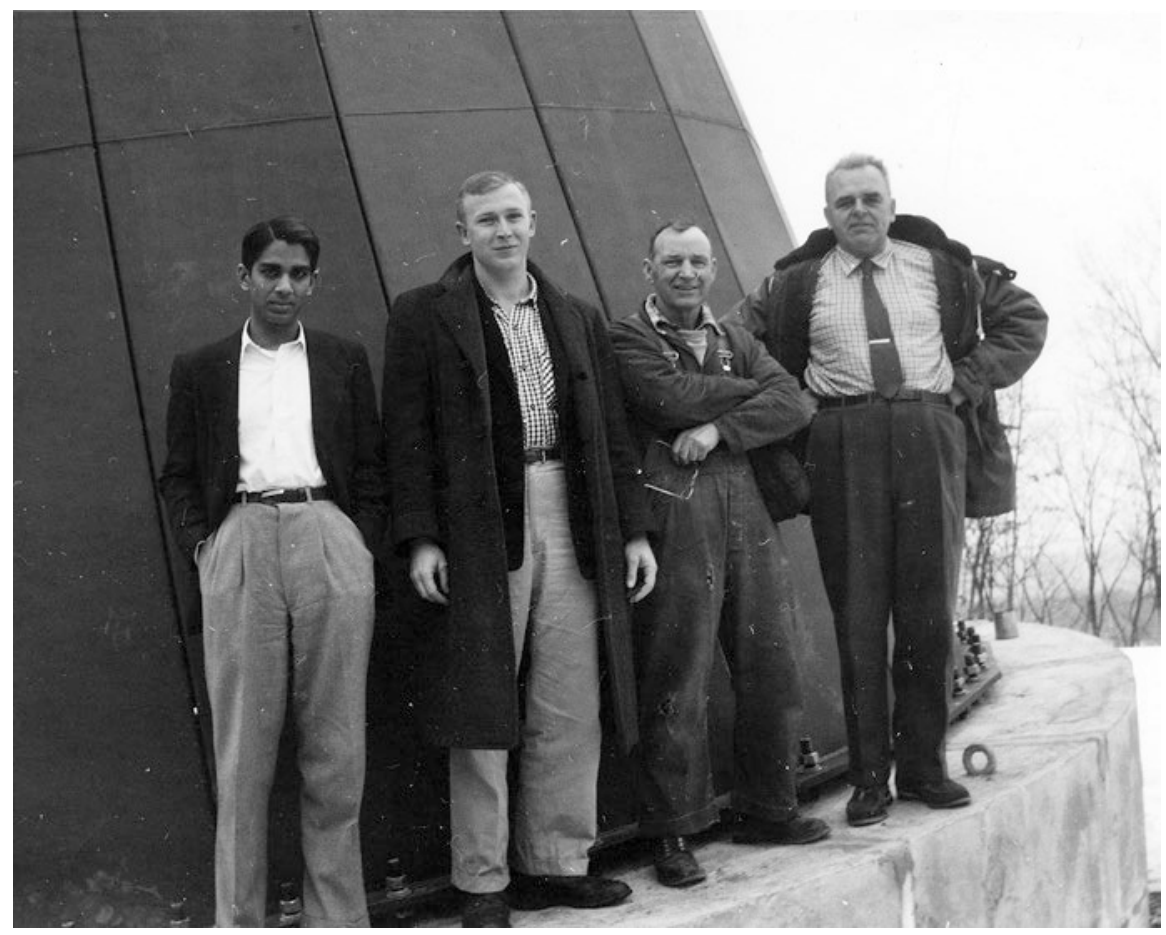

Fig. 2.3 Kochu Menon, Dave Heeschen, Russell Anderson, and Bart Bok at the base of Harvard's 60 foot Agassiz telescope in early 1956. Credit: NRAO/AUI/NSF

nearby Air Force Cambridge Research Lab, then to Berkeley where she played a key role in the discovery of cosmic hydroxyl masers (Dieter et al. 1966). Tom Matthews went to Caltech where he contributed to the discovery of quasars. John (Jack) Campbell became one of Ewen's first employees before going on to become Systems Engineer for the VLA. Cam Wade, while still a graduate student, worked for Ewen-Knight before taking up a postdoctoral position in Australia, followed by a long career on the NRAO Scientific Staff where he led the VLA site search and procurement. After a postdoc at NRAO followed by a faculty position at the State University of New York, May Kaftan-Kassim returned to her native Iraq where she became Director of the Iraqi National Astronomy Observatory Project, ${ }^{34}$ and later the Iraqi representative to the 1968 UN Vienna Conference on the Exploration and Peaceful Uses of Outer Space. After a period at NRL and Yale, Ed Lilley returned to join the Harvard faculty where he spent the rest of his career.

Cornell University The Cornell solar radio astronomy program began in the Electrical Engineering Department in the late 1940s with support from the AFOSR and ONR. Under the leadership of Charles Burrows, William (Bill) Gordon, and Charles (Charlie) Seeger, older brother of the well-known folk 
singer, Pete Seeger, this was probably the first US university based program in radio astronomy. Martha Stahr, who was a Professor of Astronomy and the first female faculty member in the Cornell School of Arts and Sciences, conducted daily observations of the Sun at $205 \mathrm{MHz}$ which she related to sunspot activity (Stahr 1949). Later the group extended their research program to include mapping the galactic radiation (Seeger and Williamson 1951). From 1948 to 1960, Stahr (later Stahr Carpenter) also issued a valuable series of bibliographic reports listing publications in radio astronomy that brought radio astronomy work, which at the time was largely published in engineering journals, to the attention of the broader astronomical community. ${ }^{35}$

After Seeger left Cornell for Sweden in 1950, then the Netherlands, Marshall Cohen, who had received his $\mathrm{PhD}$ in physics from Ohio State, joined the Cornell group in 1954 and began a program to study the polarized solar radio emission. Cohen later recalled, "What we were doing at Cornell when I got there-I found out very quickly was really quite primitive compared to what was going on in Australia." And in fact, part of the Cornell program was terminated not long afterward. ${ }^{36}$

Starting in 1958, under the leadership of Bill Gordon, Cornell scientists began discussing the construction of a 1000 foot (305 meter) diameter back scatter radar system to study the ionosphere. Cohen realized that with the proposed antenna it would also be possible to get radar echoes from Mars and Venus and pointed out other passive radio astronomy applications of the Arecibo Ionospheric Observatory (See Sect. 6.6) which was later funded by the AFOSR.

The University of California at Berkeley The radio astronomy program at the University of California began somewhat later than the other university programs, and, like the program at Harvard, was initiated and initially managed by classical astronomers with backgrounds in optics rather than radio engineering. The Berkeley Astronomy Department was rejuvenated after WWII by Otto Struve, who came to Berkley as department chair in 1950. The following year, he brought Harold Weaver to Berkeley from the Lick Observatory, and in 1954, at Weaver's invitation, Ron Bracewell came to Berkeley from the CSIRO Radiophysics Laboratory and taught a course in radio astronomy. With the urging of Otto Struve, who was perhaps reflecting on his unsuccessful effort a decade earlier to help Grote Reber get established, the Dean established a faculty committee to investigate how the university could enter the new field of radio astronomy. ${ }^{37}$ A favorable committee report provided the incentive for Otto Struve and Harold Weaver to propose a new observatory, but considerably larger than the one envisioned in the committee report. In 1958, following Weaver's sabbatical at Harvard and the Carnegie Department of Terrestrial Magnetism, Weaver and Struve found the funding from ONR needed to establish the Berkeley Radio Astronomy Laboratory. They selected a site at Hat Creek in northern California to build first a 33 foot (10 meter) radio telescope, and in 1962 an 85 foot (26 meter) telescope 
constructed by the Philco Corporation. Doc Ewen built the $1420 \mathrm{MHz}$ and $8 \mathrm{GHz}$ receivers.

Over the next three decades Weaver led a vigorous observational program concentrating on $21 \mathrm{~cm}$ galactic research, and in 1965, Hat Creek made the first observations of interstellar $\mathrm{OH}$ masers, opening a whole new field of research. In January 1991, the Hat Creek 85 foot radio telescope collapsed in a storm with 100 mile per hour winds. But by that time, under the leadership of Jack Welch, the emphasis at Hat Creek had moved to millimeter wavelengths (Sect. 10.4) and the telescope was not replaced.

Stanford University Following a one year visiting appointment at Berkeley, Ron Bracewell went to Stanford, and, with funding from AFOSR, built the Stanford Microwave Spectroheliograph. The Spectroheliograph, based on the design by Christiansen, consisted of two orthogonal arrays of sixteen 10 foot $(3 \mathrm{~m})$ dishes each 375 feet $(114 \mathrm{~m})$ long (Bracewell and Swarup 1961). Operating at $3.3 \mathrm{GHz}(9.1 \mathrm{~cm})$ the Stanford array was used until 1980 to make daily maps of the microwave emission from the solar corona with an angular resolution of $3.1 \mathrm{arcmin}$. During this period, more than 200 astronomers visited the site and engraved their names on the telescope piers. In 2012, ten of the piers were moved to the VLA site in New Mexico where they form part of an innovative sundial designed by Woody Sullivan (Frater et al. 2017, p. 1; Sullivan et al. 2019) (Fig. 2.4).

Ohio State University John Kraus was a radio engineer and an expert on antenna design who was known for his invention of the helical antenna. Also, as a prominent radio amateur, he designed the popular W8JK antenna. Kraus was present at Jansky's 1935 Detroit IRE presentation, where he recalled that there were less than two dozen people in the audience (Kraus 1984). In 1941, Kraus and Grote Reber worked together at the Naval Ordnance Laboratory degaussing naval ships, and they lived in the same rooming house in Washington. Kraus (1995, p. 114) later recalled Reber's "contagious enthusiasm," about his equipment and observations. Apparently, his exposure to these two pioneers of radio astronomy ignited Kraus' interest and excitement about this new field of research, and a decade later he initiated a radio astronomy program at Ohio State University.

Kraus was captivated by the ongoing source count controversy and the desire to push radio catalogues to weaker sources. This meant building the largest possible collecting area for a given cost, which Kraus translated into a transit radio telescope. His initial radio telescope consisted of an array of 96 helical antennas mounted on a tiltable ground plane. Over a period of three years, Ko and Kraus (1957) used this early radio telescope to map the sky north of -40 degrees declination at 1.2 meters wavelength $(250 \mathrm{MHz})$ with a resolution of 1 by 8 degrees. In addition to tracing out the radio emission from the galactic plane, the Ohio State Survey isolated a number of discrete sources, which they noted are concentrated along the galactic plan. 
Fig. 2.4 Ron Bracewell touches up paint on a pier at the Stanford Spectroheliograph with the signatures of visiting astronomers, including one of the present authors (KK). Ten of the original piers are now part of a sundial at the VLA site. Credit: NRAO/AUI/ NSF

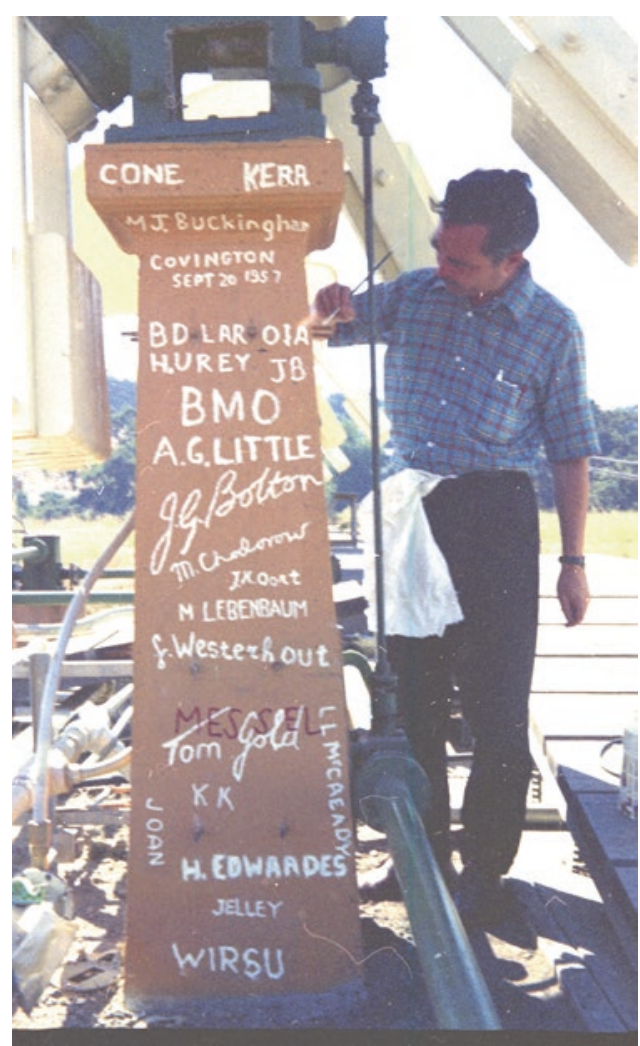

\subsection{US Government and Military Radio}

\section{Astronomy Programs}

National Bureau of Standards Grote Reber's mother died in 1945 and there was little reason for him to remain in Wheaton. He sought support from the Carnegie Institution's Department of Terrestrial Magnetism, Harvard, MIT, and various government agencies. In a 1946 letter to Harlow Shapley, Reber estimated the cost, including labor, of reproducing his telescope to be $\$ 15,278 .{ }^{38}$ At Harvard, Shapley discussed Reber's proposal with Donald Menzel, who had a background in radiophysics and had many contacts in the military industrial complex (Needell 2000, p. 60). As with Reber's earlier approach to Harvard, Shapley decided they were too busy and had insufficient resources to take on any new projects. Reber's letter stirred up interest for both Merle Tuve and Lloyd Berkner at DTM as well as their Carnegie boss, Vannever Bush. Probably inspired by Reber, Tuve started the DTM radio astronomy program led by Lloyd Berkner. A few years later, Berkner would become President of Associated Universities, Inc. (AUI), where he forcefully led the effort to establish the National Radio Astronomy Observatory, an initiative first started by Bush and Lee DuBridge (Chap. 3). 
Reber realized that he was no longer able to compete with these larger government and university activities. He accepted an offer from E.U. Condon to set up a radio astronomy program at the National Bureau of Standards (NBS) Central Radio Propagation Laboratory (CRPL) in Washington, DC, with the prospect of building a 75-100 foot diameter dish. He sold his Wheaton dish and all instrumentation, including a $1400 \mathrm{MHz}(21 \mathrm{~cm})$ amplifier and feed, to the US government for $\$ 18,570$. Everything was moved from Wheaton to Sterling, Virginia, near the location of the current Dulles Airport. His dish was reassembled on a turntable so it could be moved in azimuth as well as elevation, but there is no record that it was ever used again by Reber or by anyone else for radio astronomy. Reber's dish was later disassembled and transported to Boulder, where it remained unassembled until it was moved to Green Bank to be erected under Reber's supervision at the entrance to NRAO in 1959-1960 (Fig. 2.5).

Arguing that a 100 foot dish would only be a small improvement over his Wheaton dish, one of Reber's first actions in Washington was to develop detailed plans for building a large, fully steerable dish 220 feet in diameter operating to wavelengths as short as $10 \mathrm{~cm}(3 \mathrm{GHz})$, which he later estimated might cost $\$ 650,000$ to build. ${ }^{39}$ Realizing that an equatorial mount for an antenna of this size would be prohibitively expensive, he proposed using an alt-azimuth mount with an innovative "combination of cams and levers" to control the telescope (Fig. 2.6).

Reber approached CRPL management, the Pentagon, and Merle Tuve at Carnegie, but his ambitious initiative was either rejected or ignored. Later, Lloyd Berkner at AUI expressed interest, but on the advice of his steering committee, AUI pursued a different approach (Sect. 4.4), and Reber was never able to obtain the funds to build his 220 foot antenna. Indeed, it would be nearly another half a century before a fully steerable antenna of this size would be built in the US (Chap. 9).

While working at NBS, Reber participated in a NRL expedition to observe a total solar eclipse on the island of Attu, at the westernmost end of the Aleutian Islands. Multi-wavelength observations on 12 September 1950 showed that the radio Sun was larger than the optical disk, and that the apparent size increased with increasing wavelength (Hagen et al. 1951). As Reber later reported, this "was probably the first total solar eclipse of the Sun which was successfully observed in a pouring rain during a hurricane." ${ }^{40}$

Naval Research Laboratory 50 Foot Radio Telescope ${ }^{41}$ Perhaps the biggest organized postwar radio astronomy program in the United States, or at least the most expensive, was at NRL, which began as an outgrowth of wartime radar and communications research. The operation of NRL is led by a Naval Captain, but the research program is led by a civilian. Early NRL postwar research programs concentrated on the Sun, partly because the military was interested in the Sun and how it affected communications, but perhaps, like elsewhere, more because that was all they could see with their limited 


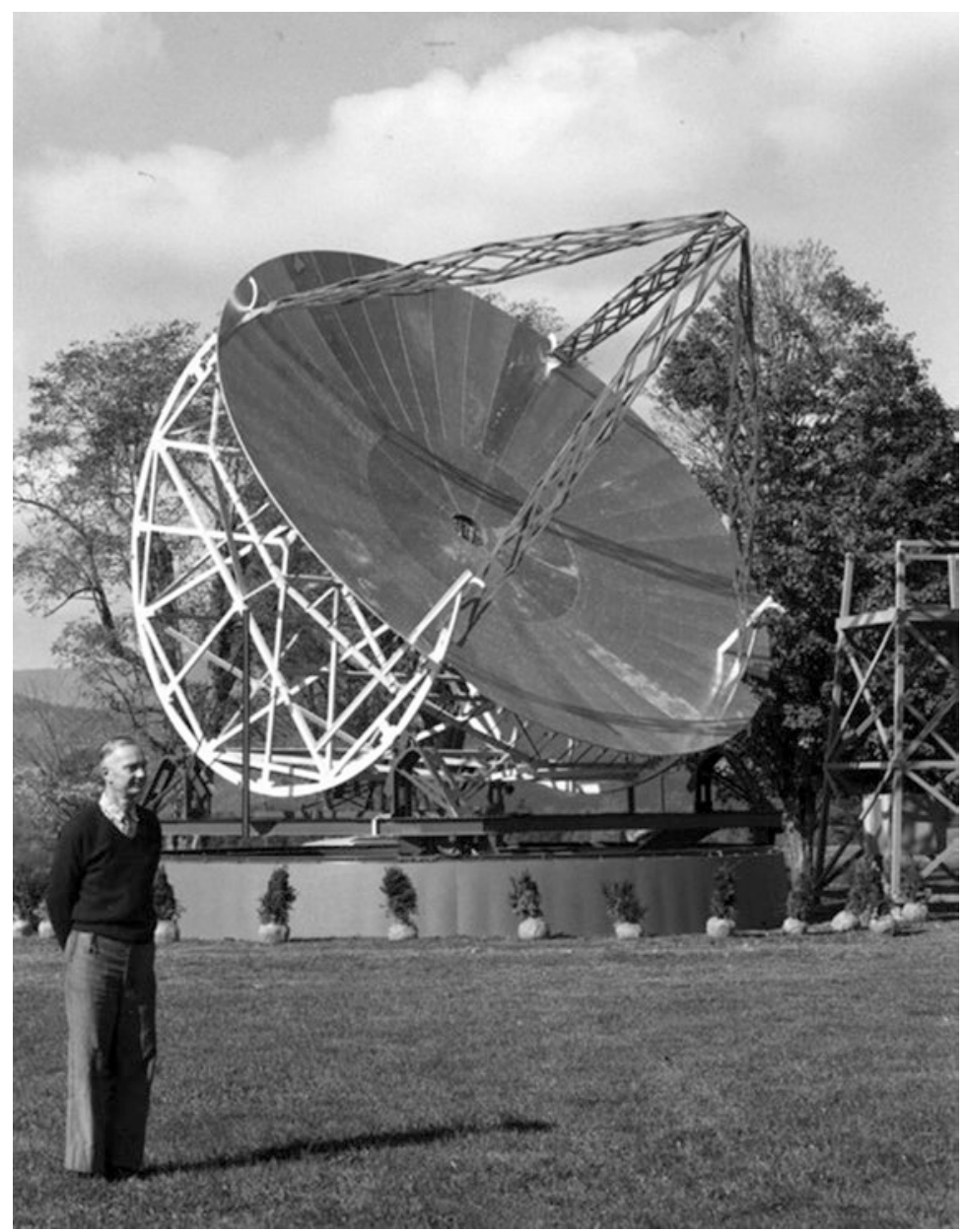

Fig. 2.5 Grote Reber in 1960 beside his reconstructed 32 foot Wheaton radio telescope at the entrance to NRAO in Green Bank. Credit: NRAO/AUI/NSF

equipment. In 1951, under the leadership of John Hagen, who had led a wartime NRL centimeter wavelength radar development program, NRL obtained a 50 foot ( 15 meter) antenna from the Collins Radio Company. The antenna was designed by University of Iowa engineering professor Ned Ashton, whose previous experience was primarily in designing bridges. Ashton would go on to play a major role in the troubled NRAO 140 Foot Telescope project (Sect. 4.4). With a background in radio engineering rather than astronomy, NRL chose an alt-az mount for its radio telescope, using a surplus five inch gun mount for the azimuth bearing and an analogue coordinate converter to provide pointing instructions. But the 1.5 arcminute pointing accuracy made operation difficult at $8 \mathrm{~mm}$, where the beamwidth was only 3 arcminutes, and 


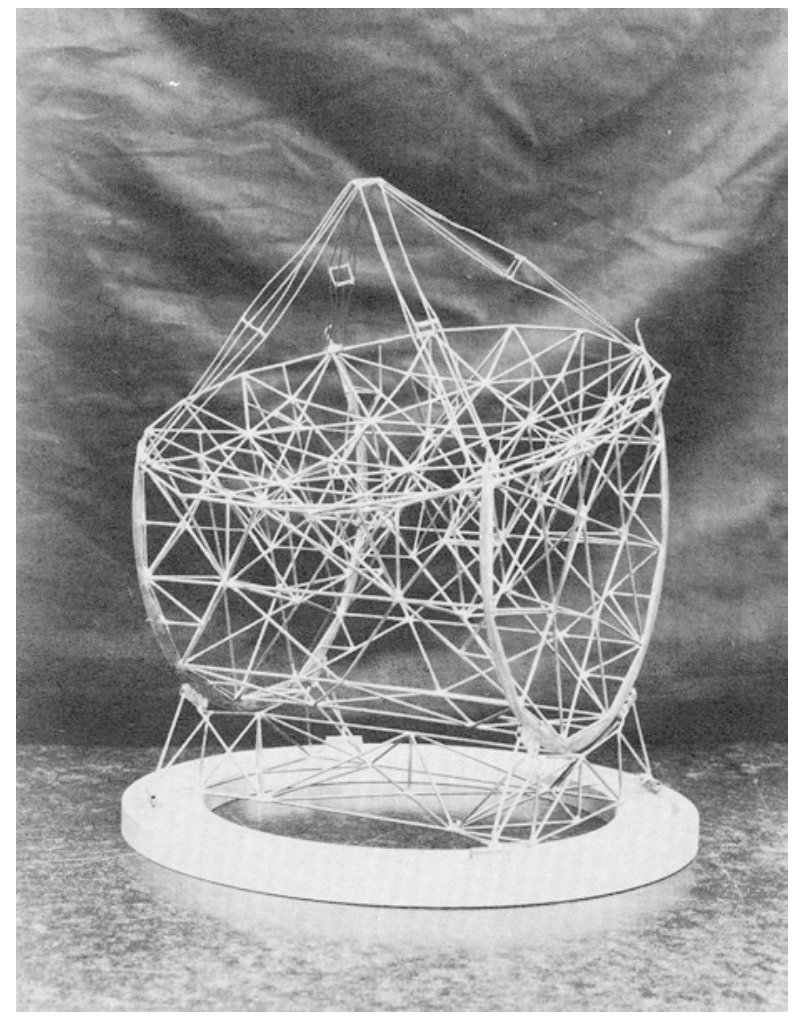

Fig. 2.6 Grote Reber's model for a 220 foot telescope design. Credit: Feld, J. Radio Telescope Structures. 1962. Ann. NY Acad. Sci. 93: 353-456. Used with permission of the NYAS

gave alt-az mounts a bad name during later debates over the nature of NRAO's 140 Foot Telescope. The machined surface, which was composed of 30 separate solid aluminum panels, had a maximum deviation from a true paraboloid of $0.8 \mathrm{~mm}$ in the inner 20 foot diameter and $1.2 \mathrm{~mm}$ in the outer part of the dish (Holzschuh 1958).

When completed in 1951, the NRL antenna (Fig. 2.7) was the largest filled aperture radio telescope in the world, and would remain so until the Harvard 60 foot and the 25 meter Dwingeloo dish went into operation four years later (Fig. 2.8). However, working primarily at short centimeter wavelengths, the sensitivity and research opportunities were limited. As Graham-Smith remarked to Joe Pawsey "it was the world's most expensive radio telescope and all it can see is the Sun and the Moon" (Haddock 1984). Led by Fred Haddock and later Ed McClain and Cornell Mayer, the 50 foot telescope was used by NRL scientists at $9.4 \mathrm{~cm}(3.1 \mathrm{GHz})$ to study the thermal radio emission from galactic H II regions and the planets Venus (Mayer et al. 1958a), Mars, and Jupiter (Mayer et al. 1958b), to make the first observations below $21 \mathrm{~cm}$ of a few 
strong radio galaxies (Haddock et al. 1954), and the first measurement of radio polarization (Mayer et al. 1957). NRL also pioneered $21 \mathrm{~cm}$ absorption line studies (Hagen and McClain 1954). However, in what proved to be an embarrassment, Lilley and McClain (1956) reported the detection of the redshifted $21 \mathrm{~cm}$ absorption line from Cygnus A which not only was never confirmed by others, but it turned out Lilley and McClain had incorrectly used the optical redshift to calculate the radio frequency of the expected absorption and were observing at the wrong frequency.

As it was at the time the most important and the most influential American radio astronomy observatory, NRL scientists, particularly John Hagen, had considerable influence on the later formation of NRAO (Chap. 3). The NRL telescope still sits on its original location on the roof of a laboratory building overlooking the Potomac River. For more than half a century it served as an NRL icon, visible from commercial aircraft landing over the Potomac River at nearby Reagan National Airport.

The location of the NRL dish in Washington and near National Airport meant it was subject to considerable RFI; NRL needed to find a new site for radio astronomy. In 1955, NRL obtained an 84 foot (26 meter) antenna from the D.S. Kennedy Company, patterned after Harvard's Kennedy-built 60 foot radio telescope (McClain 1958). The site for the 84 foot dish was chosen following a survey using the same equipment that had been used in 1955 to

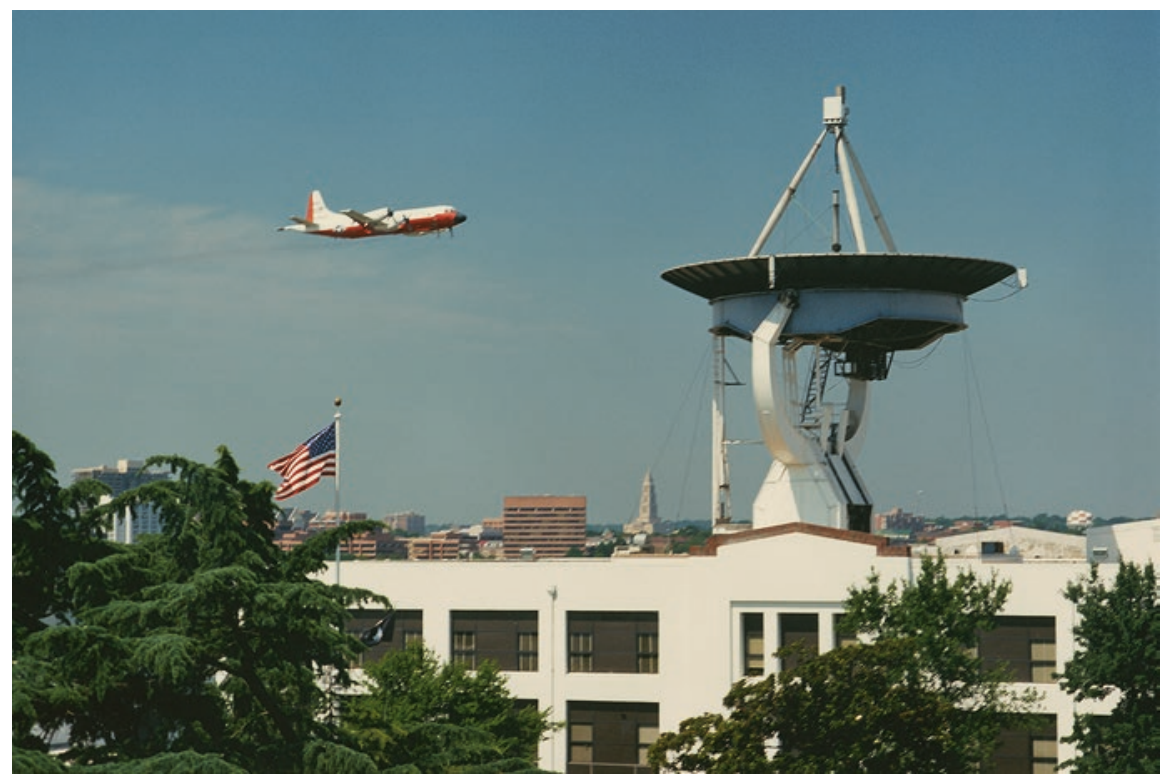

Fig. 2.7 NRL's iconic 50 foot radio telescope on the roof of the main laboratory building, visible from commercial flights into and out of nearby Reagan National Airport. Credit: NRL 


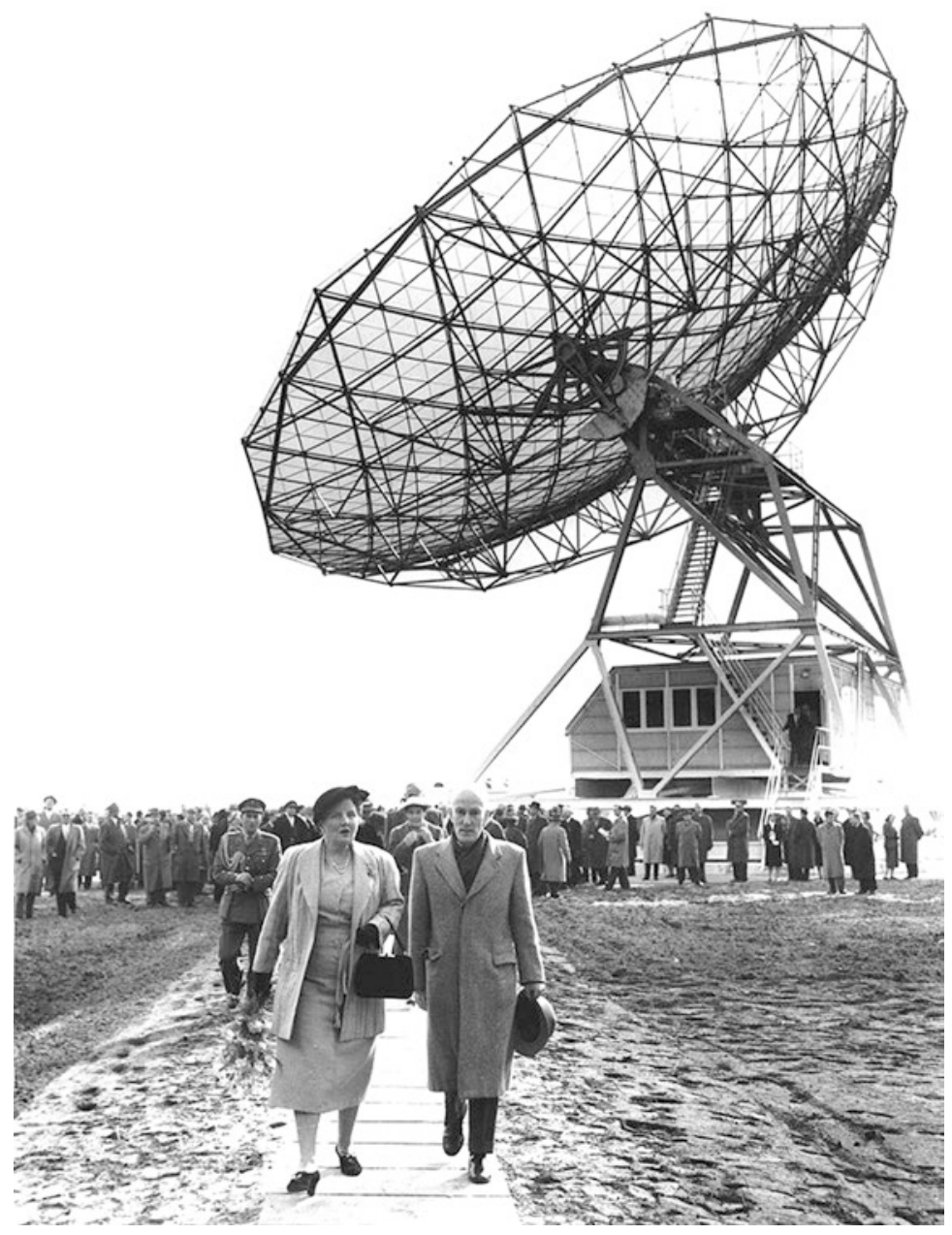

Fig. 2.8 Prof. Jan Oort and Queen Juliana leaving the 1956 dedication of the Dwingeloo 25 meter radio telescope. Credit: NFRA

locate the Green Bank site (Sect. 3.4). The chosen site at Maryland Point, Maryland, about 45 miles from the NRL Washington Lab, was found to be the quietest site within 50 miles of Washington. The Kennedy dish had a perforated aluminum surface and was effective at wavelengths as short as $10 \mathrm{~cm}$.

\subsection{Private Initiatives}

Carnegie Institution Department of Terrestrial Magnetism (DTM) Shortly after the end of WWII, Lloyd Berkner and Merle Tuve initiated a modest program in radio astronomy at DTM. According to Burke (2003), Tuve rejected 
Berkner's ambitious plan to build a large dipole array, probably contributing to the long-running animosity between Tuve and Berkner that would soon impact Berkner's plans to develop a national radio astronomy facility (Chap. 3). As explained in Sect. 2.4, before Bok and his students got involved in $21 \mathrm{~cm}$ research, Tuve had talked Purcell into sending him Ewen's $21 \mathrm{~cm}$ receiver, which he initially used on a German Würzburg. DTM's Howard E. Tatel designed an equatorial mount for the 7.5 meter telescope, making it unlike the Würzburgs used in European radio observatories.

In 1953, Tuve was joined by Bernard Burke, a recent graduate from MIT, and by F. Graham-Smith, on a one-year leave from Cambridge. Together, Graham-Smith and Burke built a $22 \mathrm{MHz}$ Mills Cross at a site in Maryland. After Graham-Smith returned to Cambridge, Burke, working with Ken Franklin, who was on leave from New York's Hayden Planetarium, accidently discovered the strong $22 \mathrm{MHz}$ bursts from Jupiter (Burke and Franklin 1955). In 1959 DTM purchased a 60 foot (18 meter) dish from Blaw-Knox, which was used by Tuve and others for mapping the distribution of galactic hydrogen.

Grote Reber Goes to Hawaii ${ }^{42}$ After only a few years working at the NBS, Grote Reber became discouraged by the lack of support for his planned large radio telescope. He had also become increasingly frustrated working under government bureaucracy, as well as by the deteriorating atmosphere in Washington reflected by the growing impact of McCarthyism. He was intrigued by the discoveries reported by Australian radio astronomers using the sea interferometer technique (Sect. 2.1) which could determine the positions of radio sources from the precise timing as they rose or set over the sea. He realized that in order to obtain the accurate two-dimensional coordinates needed for identification with optical counterparts, he would need to make measurements at both rising and setting, and that the best place to do this was from a mountaintop in Hawaii. In a letter to Joe Pawsey, Reber wrote "I got tired of working for Uncle Harry [Truman] and his Boys so I took a vacation in Hawaii. Things looked so good I decided to stay." ${ }^{43}$ He went on vacation to Hawaii, and never came back to NBS, apparently abandoning his last paycheck.

From 1951 to 1954 , Reber worked on top of Haleakala on the Island of Maui, Hawaii, where he built a rotating antenna to observe between 20 and $100 \mathrm{MHz}$. By replacing the fixed antenna used by the Australians with one that rotated in azimuth, and by working on the top of a 3000 meter high island mountain, he could observe sources both rising in the east and setting in the west with an interferometer having an effective baseline of 6000 meters. In principle his sea interferometer had a resolution of about one arcminute, but he was plagued by ionospheric refraction and terrestrial interference and was only able to obtain useful results on a few of the strongest radio sources (Reber $1955,1959)$. As a result of his experience, he finally concluded that mountain tops were not suitable for radio telescopes. But Reber's sea interferometer was the first mountain-top telescope in the Hawaiian Islands and set the stage for 
the proliferation of optical and radio telescopes later constructed on both Haleakela and Mauna Kea. The site of Reber's radio telescope was preserved until 2014, when it was destroyed to make room for a parking lot for the Daniel K. Inouye Solar Telescope.

\subsection{Why Did the US Fall Behind the UK and Australia? Or Did IT?}

Looking back after more than half a century, it is natural to ask-did the United States really fall behind the radio astronomy programs being pursued elsewhere even in other, much smaller countries such as the Netherlands and Australia? Or, did American radio astronomers just move in a different direction, or just imagine that they were behind, or perhaps even exaggerate the situation in an attempt to acquire more resources? In the UK, Australia, and the Netherlands, radio astronomy programs were generally concentrated at one or two major laboratories. In both Australia and the UK, early postwar radio astronomy research was conducted primarily at meter wavelengths, using relatively simple and relatively inexpensive technology with equipment derived from wartime radar research. In the United States, reflecting the broad enthusiasm to get into this exciting and promising new field of research, modest radio astronomy programs sprang up at a few universities, but primarily at government and military laboratories. The early postwar US radio astronomy programs were mostly funded by the military, and so were driven, at least in part, by Cold War military needs. This translated into facilities that operated at centimeter wavelengths where there was considerable expertise and experience carried over from the War, but where receiver sensitivities were much poorer than at the meter wavelengths used in the UK and Australia, and where the cost and the complexity of antennas and instrumentation were greater.

At least for the first decade following the end of WWII, there was no focused effort in the US to develop any major radio astronomy research programs of the type being pursued at Cambridge and Jodrell Bank or in Sydney or Leiden. The US entered the postwar era with relatively great wealth, especially when compared with Australia or war-torn Europe. So why did this new field that had been pioneered by the Americans Karl Jansky and Grote Reber appear to thrive in the UK and Australia, but not in the US?

In part, the answer may lie in the enormous prestige of postwar American science and scientists, brought about by the very successful atomic energy program which had led to an abrupt and unanticipated, but greatly welcomed, end to the war in the Pacific. The related areas of atomic and nuclear physics overshadowed the equally important-some will say more important-developments in radiophysics and electronics resulting from the wartime radar research at the MIT Radiation Laboratory, Bell Labs, and elsewhere. Although, as Lee DuBridge remarked, "the bomb ended the war, but radar won the war," 44 atomic and nuclear physics became the golden areas of research for American 
scientists, with a seemingly endless flow of money leading to powerful particle accelerators being built at Harvard, the University of California, Caltech, Cornell, Rochester, and other universities, as well at the new Brookhaven National Laboratory.

At the end of 1945, the MIT Rad Lab was shut down. Robert Dicke (Princeton), Lee DuBridge (Rochester), E.O. Lawrence (Berkeley), Edwin McMillan (Berkeley), Ed Purcell (Harvard), and I.I. Rabi (Columbia), all left for universities where they applied their skills in microwave electronics to atomic spectroscopy and particle accelerator physics. Charlie Townes, who was one of the first to recognize the possibility of observing cosmic molecular lines in the radio spectrum (Townes 1957), moved to Columbia University, where he worked on quantum electronics and the development of the maser and later the laser. Taffy Bowen, the Rad Lab liaison to the British radar program, left for Australia, where he started the very successful CSIRO radio astronomy program.

For nearly a decade, almost no one in the US paid much attention to radio astronomy after Jansky and Reber and the casual experiments of Potapenko and Folland. Reber, in particular, was unable to obtain funding or even recognition of his ambitious plan to build a 220 foot dish for radio astronomy, and he ultimately left the United States for Tasmania, where he carried out a program in long wavelength radio astronomy as well as publishing papers in botany and archaeology. NRL did build the then largest steerable radio telescope in the world, and one that operated at much shorter wavelengths than any other contemporary radio telescope, but the motivation was more to address military needs than astronomy. The NRL 50 foot dish also pioneered the use of the alt-az mount in astronomy but, working at short centimeter and millimeter wavelengths, the scientific returns were perceived to be limited.

Much good research was done by US radio astronomers. Berkeley, Cornell, Harvard, Ohio State, and Stanford started important university programs in solar radio astronomy. NRL made the first detailed studies of the thermal radio emission from the Moon and planets. Franklin and Burke at DTM discovered intense radio bursts from Jupiter. Ewen and Purcell at Harvard were the first to detect the $21 \mathrm{~cm}$ hydrogen line, beating the Australian and Dutch radio astronomers and starting the new field of cosmic radio spectroscopy. Ewen and Purcell also invented the important frequency switched spectral line radiometer, following Robert Dicke's invention of the switched radiometer, since used by generations of radio astronomers for continuum observations. However, none of these programs captured the attention of the US astronomy community as did the Australian and British discoveries of supernova remnants and distant powerful radio galaxies. As it developed, the cosmological Cambridge-Sydney $\log N-\log S$ debate was a red herring, full of observational errors and naive interpretation on both sides, but for over a decade, the apparent impact to cosmology captivated the astronomical community. 
The discovery of the $21 \mathrm{~cm}$ hydrogen line by Ewen and Purcell at Harvard did create a unique opportunity, but one that was exploited first by the Dutch and Australian radio astronomers in mapping the dynamics of the Milky Way. While Purcell continued his research on nuclear magnetic resonance, Ewen went into business, manufacturing and selling radio astronomy receivers. Bart Bok built the Harvard radio astronomy project around $21 \mathrm{~cm}$ research, but the Australian and Dutch radio astronomers skimmed the cream, and the Harvard program suffered from lack of leadership after Bok went to Australia. However, Bok did develop what was probably the first graduate program in radio astronomy, a program from which many of the first generation of students went on to lead the establishment of the National Radio Astronomy Observatory in Green Bank.

\section{Notes}

1. This is equivalent to the 1834 classical Lloyd's mirror optical effect.

2. Because the signals from different parts of the radio source have slightly different path lengths, the interferometer response is sensitive to source dimensions. The paper by McCready et al. was apparently submitted with Pawsey as the first author but the order was changed by the editor. We thank Ron Ekers and Miller Goss for bringing this historical note to our attention.

3. A more extensive discussion of the various discoveries of solar radio emission is given by Sullivan (2009) pp. 79-99, and for later observations, pp. 284-314.

4. In 1945, Elizabeth Alexander detected radio emission from the Sun while operating a $200 \mathrm{MHz}(150 \mathrm{~m})$ radar on Norfolk Island in the South Pacific, as described in her "Report on the Investigation of the Norfolk Island Effect," NAA-WTS, Working Papers, E. Alexander. See also Orchiston (2005).

5. A copy of Hey's 1942 secret report is located in NAA-WTS, Sullivan Publications, Classics in Radio Astronomy.

6. Even the published results in $\mathbf{1 9 4 5}$ were devoid of any technical details including the observing frequencies and antenna.

7. GR to JLG, 3 November 1946, NAA-GR, General Correspondence. https:// science.nrao.edu/about/publications/open-skies\#section-2

8. GR to JLG, 21 November 1946, NAA-GR, General Correspondence I. https:// science.nrao.edu/about/publications/open-skies\#section-2

9. In this Nature letter, which carries a submission date of 24 November, Reber reports on observations made on 23 and 24 November. No year is given anywhere, but presumably all dates refer to 1946 . The paper was published in the 28 December 1946 edition of Nature.

10. The ionosphere introduces apparent fluctuations in the intensity of small radio sources in much the same way as the atmosphere causes the twinkling of stars. Since telescopes more than a hundred or so miles apart see a different ionosphere the fluctuations appear uncorrelated. If the variations were intrinsic, they would appear the same at different sites.

11. Interview with KIK, August 1989. 
12. In a static universe with normal Euclidean geometry, since the number of sources, $N$, within a given distance, $D$, is proportional to the volume, $D^{3}$, while the flux density, $S$, is related to $1 / D^{2}$, it follows that $N$ is proportional to $S^{-3 / 2}$ or $\log N={ }^{-1} .5 \log S$. Thus when plotted on a $\log -\log$ plot of number vs. flux density, the expected slope is -1.5 .

13. To address what he saw as a legitimate criticism of the $2 \mathrm{C}$ catalog, Scheuer (1957) developed a statistical analysis of the interferometer data which was not subject to the errors of noise and confusion that contributed to cataloging individual sources. Hewish (1961) used Scheuer's method to calculate a slope of - 1.8 for the new 4C survey, in good agreement with the value obtained from counting individual sources.

14. We now know that indeed there are structures in the distribution of galaxies on scales of hundreds of Megaparsecs.

15. Crawford, Jauncey, and Murdoch (1970) showed how to derive the differential source count from ungrouped data.

16. See also the Nobel Prize lectures by Adam Riess, https://www.nobelprize.org/ prizes/physics/2011/riess/lecture/, Brian Schmidt, www.nobelprize.org/ prizes/physics/2011/schmidt/lecture/, and Saul Perlmutter, https://www. nobelprize.org/prizes/physics/2011/perlmutter/lecture/

17. Modern radio spectroscopy typically employs narrow band filters to separate the narrow band radiation from atomic and molecular transitions, which are referred to as "spectral lines," by analogy with optical Fraunhofer lines observed in solar and stellar visual spectra.

18. Radio recombination lines (RRL) are an extension of the familiar Lyman and Balmer series in the optical spectrum, but with quantum numbers much larger than 1. The detection of RRLs is discussed further in Chap. 6. The $1420 \mathrm{MHz}$ $(21 \mathrm{~cm})$ hyperfine transition occurs when the electron and proton spins flip from being in the same direction to opposite directions with a release of energy, corresponding to $E / h=1420.404 \mathrm{MHz}$. where $h$ is the Planck constant.

19. J. Oort to GR, 30 August 1945. NAA-GR, General Correspondence I. https:// science.nrao.edu/about/publications/open-skies\#section-2

20. GR to JLG, 19 November 1950, NAA-GR, General Correspondence I. https:// science.nrao.edu/about/publications/open-skies\#section-2

21. A personal account of Ewen's career and his detection of the $21 \mathrm{~cm}$ line can be found in https://science.nrao.edu/about/publications/open-skies\#section-2, his interview with the AIP at https://www.aip.org/history-programs/nielsbohr-library/oral-histories/6659, his interview with Woody Sullivan at, https://science.nrao.edu/about/publications/open-skies\#section-2, and Ewen's 19 February 1978 handwritten notes to Purcell held at the Harvard University Archives in the papers of Edward Purcell.

22. Ewen to Purcell, 19 February 1978, op. cit.

23. In order to mitigate the effect of receiver instabilities, Ewen's radiometer recorded the difference between two nearby frequencies. However, his initial choice of only $10 \mathrm{kHz}$ between the signal and reference frequencies proved to be too close, and so most of the galactic $21 \mathrm{~cm}$ signal was canceled out.

24. H. Ewen to E. Purcell, 2 February 1978, op. cit.

25. https://science.nrao.edu/about/publications/open-skies\#section-2

26. H. Ewen to E. Purcell, 2 February 1978, op. cit. 
27. These two reports, The Radio-Frequency Line-Spectrum of Atomic Hydrogen, I. The Calculation of Frequencies of Possible Transitions, and 2. The Calculation of Transition Probabilities were distributed as RPL 33 (February 1949) and RPL 34 (May 1949) respectively. It is not clear if these papers were known to Ewen and Purcell or to Oort and van de Hulst, but were surely known to Wild's Radiophysics colleagues Bowen and Pawsey.

28. GR to H. Ewen and E. Purcell, 8 October 1951, NAA-GR, General Correspondence I. https://science.nrao.edu/about/publications/openskies\#section-2. The $327 \mathrm{MHz}$ line of deuterium escaped detection by radio astronomers for more than another half century (Rogers 2007).

29. https://science.nrao.edu/about/publications/open-skies\#section-2

30. The initiative by Wade to involve Bok and Ewen in pursuing further radio astronomy work at Harvard was described by Wade, T.K. Menon and David Heeschen in their interviews with the authors 21 March 2015, 27 July 2012, and 31 May 2011 respectively, NAA-KIK, Oral Interviews. https://science. nrao.edu/about/publications/open-skies\#section-2

31. Probably the first PhD based on work done in radio astronomy was John Hagen, who received his $\mathrm{PhD}$ in 1949 based on a observations of solar radio emission.

32. Only the 25 meter (82 foot) Dwingeloo Telescope completed in 1955 was larger.

33. A. Waterman, 28 April 1956, "Windows on the Future," Remarks at the Dedication of the George A. Agassiz Telescope. NAA-NRAO, Founding and Organization, Planning Documents. https://science.nrao.edu/about/publications/open-skies\#section-2

34. The partially built 30 meter Iraqi radio telescope was destroyed in a bombing attack by the Iranian Air Force.

35. See Bibliography of Radio Astronomy, Bibliography of Extraterrestrial Radio Noise, and Bibliography of Natural Radio Emission from Astronomical Sources.

36. Cohen, Marshall H. Interview by Shelley Erwin. 1996, 1997, 1999, Oral History Project, Caltech Archives. http://resolver.caltech.edu/CaltechOH: OH_Cohen_M

37. Committee members included the physicist Luis Alvarez, Sam Silver from Electrical Engineering, and Harold Weaver from Astronomy.

38. GR to H. Shapley, 27 July 1946, NAA-GR, General Correspondence I. https:// science.nrao.edu/about/publications/open-skies\#section-2

39. GR to O. Struve, 16 July 1946; GR to H. Shapley, 27 July 1946; GR to C. Schauer, 12 October 1954, NAA-GR, General Correspondence I. https:// science.nrao.edu/about/publications/open-skies\#section-2

40. GR to O. Struve, 3 October 1950, NAA-GR, General Correspondence I. https://science.nrao.edu/about/publications/open-skies\#section-2

41. A detailed description of the NRL 50 foot dish is given by Holzschuh (1958).

42. This section on Grote Reber is adapted from Kellermann (2004), with permission from the Astronomical Society of the Pacific.

43. GR to J. Pawsey, 19 December 1951, National Archives of Australia. We are indebted to Miller Goss for bringing this letter to our attention.

44. Quoted in Rabi, Citizen and Scientist (Rigden 1987), p. 164. 


\section{BIBLIOGRAPHY}

\section{REFERENCES}

Baade, W. and Minkowski, R. 1954, Identification of the Radio Sources in Cassiopeia, Cygnus A, and Puppis A, ApJ, 119, 206

Bennett, A.S. 1962, The Revised 3C Catalogue of Radio Sources, Mem. Roy. Astr. Soc., $\mathbf{5 8}, 163$

Bolton, J.G. 1948, Discrete Sources of Galactic Radio Frequency Noise, Nature, 162, 141

Bolton, J.G. 1982, Radio Astronomy at Dover Heights, Proc. Astron. Soc. Austr., 4, 349

Bolton, J.G. and Stanley, G.J. 1948, Variable Source of Radio Frequency Radiation in the Constellation of Cygnus, Nature, 161, 312

Bolton, J.G., Stanley, G.S., and Slee, O.B. 1949, Positions of Three Sources of Galactic Radio-Frequency Radiation, Nature, 164, 101

Bowen, E.G. 1984, The Origins of Radio Astronomy in Australia. In The Early Years of Radio Astronomy, ed. W.T. Sullivan III (Cambridge: CUP), 85

Bowen, E.G. 1987, Radar Days (Bristol: A. Hilger)

Bracewell, R.N. 2005, Radio Astronomy at Stanford, JAHH, 8, 75

Bracewell, R. and Swarup, G. 1961, The Stanford Microwave Spectroheliograph Antenna, a Microsteradian Pencil Beam Antenna, IRE Trans. Ant. Prop., AP-9, 22

Burke, B.F. 2003, Early Years of Radio Astronomy in the U.S. In Radio Astronomy from Karl Jansky to Microjansky, ed. L.I. Gurvits, S. Frey, and S. Rawlings (Les Ulis: EDP Sciences), 27

Burke, B.F. and Franklin, K.L. 1955, Observations of a Variable Radio Source Associated with the Planet Jupiter, JGR, 60, 213

Christiansen, W.N. 1984, The First Decade of Solar Radio Astronomy in Australia. In The Early Years of Radio Astronomy, ed. W.T. Sullivan III (Cambridge: CUP), 113

Christiansen, W.N. and Hindman, J.V. 1952a, $21 \mathrm{~cm}$ Line Radiation from Galactic Hydrogen, Observatory, 72, 149

Christiansen, W.N. and Hindman, J.V. 1952b, A Preliminary Survey of $1420 \mathrm{Mc} / \mathrm{s}$. Line Emission from Galactic Hydrogen, Austr. J. Sci. Res., A5, 437

Crawford, D.F., Jauncey, D.L., and Murdoch, H.S. 1970, Maximum-Likelihood Estimation of the Slope from Number-Flux Density Counts of Radio Sources, $A p J, 162,405$

Denisse, J.F. 1984, The Early Years of Radio Astronomy in France. In The Early Years of Radio Astronomy, ed. W.T. Sullivan III (Cambridge: CUP), 303

Denisse, J.-F., Leroux, E. and Steinberg, J.-L. 1955, Observations du Rayonnement Galactique sur la Longueur d'onde de $33 \mathrm{~cm}$, Comptes Rendus, 240, 278

Dicke, R.H. 1946, The Measurement of Thermal Radiation at Microwave Frequencies, Rev. Sci. Instrum., 17, 268

Dicke, R.H. and Beringer, R. 1946, Microwave Radiation from the Sun and the Moon, ApJ, 103, 375

Dicke, R.H. et al. 1946, Atmospheric Absorption Measurements with a Microwave Radiometer, Phys. Rev. 70, 340

Dieter, N.H., Weaver, H., and Williams, D.R.W. 1966, Secular Variations in the RadioFrequency Emission of $\mathrm{OH}, A J, 71,160$

Edge, D.O. 1984, Styles of Research in Three Radio Astronomy Groups. In The Early Years of Radio Astronomy, ed. W.T. Sullivan III (Cambridge: CUP), 351 
Edge, D.O. et al. 1959, A Survey of Radio Sources at a Frequency of $157 \mathrm{Mc} / \mathrm{s}, \mathrm{Mem}$. Roy. Astr. Soc., 68, 37

Edge, D.O. and Mulkay, M.J. 1976, Astronomy Transformed: The Emergence of Radio Astronomy in Britain (New York: Wiley)

Elbers, A. 2017, The Rise of Radio Astronomy in the Netherlands (Cham: Springer Nature)

Ewen, H.I. and Purcell, E.M. 1951a, Radiation from Hyperfine Levels of Interstellar Hydrogen, Phys. Rev., 83, 881

Ewen, H.I. and Purcell, E.M. 1951b, Observation of a Line in the Galactic Radio Spectrum, Nature, 168, 356

Frater, R.H. and Ekers, R.D. 2012, John Paul Wild AC CBE FAA FTSE. 17 May 1923-10 May 2008, Biog. Mem. R. Soc. London, 10, 229 https://royalsocietypublishing.org/doi/pdf/10.1098/rsbm.2012.0034

Frater, R.H., Goss, W.M., and Wendt, H.W. 2017, Four Pillars of Radio Astronomy, Mills, Christiansen, Wild, Bracewell (Cham: Springer Nature)

Ginzburg, V.L. 1984, Remarks on My Work in Radio Astronomy. In The Early Years of Radio Astronomy, ed. W.T. Sullivan III (Cambridge: CUP), 289

Graham-Smith, F. 1951, An Accurate Determination of the Positions of Four Radio Stars, Nature, 168, 555

Graham-Smith, F. 2005, The Early History of Radio Astronomy in Europe. In Radio Astronomy from Karl Jansky to Microjansky, ed. L.I. Gurvits, S. Frey, and S. Rawlings (Les Ulis: EDP Sciences), 1

Haddock, F.T. 1984, US Radio Astronomy Following World War II. In Serendipitous Discoveries in Radio Astronomy, ed. K.I. Kellermann and B. Sheets (Green Bank: NRAO/AUI), 115

Haddock, F.T., Mayer, C.H., and Sloanaker, R.M. 1954, Radio Emission from the Orion Nebula and Other Sources at $\lambda 9.4 \mathrm{~cm}, A p J, 119,456$

Hagen, J.P., Haddock, F.T., and Reber, G. 1951, NRL Aleutian Radio Eclipse Expedition, $S \ll T, 10(5), 111$

Hagen, J.P. and McClain, E.F. 1954, Galactic Absorption of Radio Waves, ApJ, 120, 368 Hanbury Brown, R. 1991, Boffin (Bristol: A. Hilger)

Hanbury Brown, R. and Hazard, C. 1951, Radio Emission from the Andromeda Nebula, MNRAS, 111, 357

Hanbury Brown, R. and Hazard, C. 1953, A Survey of 23 Localized Sources in the Northern Hemisphere, MNRAS, 113, 123

Heightman, D.W. 1936, Observations on the Ultra High Frequencies, TerR Bulletin, May 1937, 496

Hewish, A. 1961, Extrapolation of the Number-Flux Density Relation of Radio Stars by Scheuer's Statistical Method, MNRAS, 123, 167

Hey, J.S. 1946, Solar Radiations in the 4-6 Metre Radio Wave-length Band, Nature, 157, 47

Hey, J.S. 1973, The Evolution of Radio Astronomy (New York: Science History Publications)

Hey, J.S., Parsons, S.J., and Phillips, J.W. 1946a, Fluctuations in Cosmic Radiation at Radio Frequencies, Nature, 158, 234

Hey, J.S., Phillips, J.W., and Parsons, S.J. 1946b, Cosmic Radiations at 5 Metre Wavelength, Nature, 157, 296

Holzschuh, D.L. 1958, The NRL Precision “Big Dish” Antenna, IRE International Convention Record, 32 
Ishiguro, M. et al. 2012, Highlighting the History of Japanese Radio Astronomy. 1: An Introduction, $J A H H, \mathbf{1 5}, 213$

Kellermann, K.I. 1996, John Gatenby Bolton (1922-1993), PASP, 108, 729

Kellermann, K.I. 2004, Grote Reber (1911-2002), PASP, 116, 703 (https://doi. org/10.1086/423436)

Kellermann, K.I. ed., 2012, A Brief History of Radio Astronomy (Dordrecht: Springer), (translation of Russian edition, ed. S.Y. Braude et al.)

Kellermann, K. I. and Sheets, B. eds. 1984, Serendipitous Discoveries in Radio Astronomy (Green Bank: NRAO/AUI)

Ko, H.C. and Kraus, J.D., 1957, A Radio Map of the Sky at 1.2 Meters, SerT, $16(4), 160$

Kragh, H. 1996, Cosmology and Controversy: the Historical Development of Two Theories (Princeton: Princeton Univ. Press)

Kraus, J.D. 1984, Karl Guthe Jansky's Serendipity, Its Impact on Astronomy and Its Lessons for the Future. In Serendipitous Discoveries in Radio Astronomy, ed. K. I. Kellermann and B. Sheets (Green Bank: NRAO/AUI), 57

Kraus, J.D. 1995, Big Ear Two (Powell, Ohio: Cygnus-Quasar Books)

Kusch, P. and Prodell, A.G. 1950, On the Hyperfine Structure of Hydrogen and Deuterium, Phys. Rev., 79, 1009

Lequeux, J. 1962, Mesures Interférométriques a Haute Résolution du Diamétre et de la Structure des Principales Radiosources, Annales D'Astrophysique, 25, 221

Lequeux, J. et al. 1959, L'intérférométriques à Deux Antenna à Espacement Variable de la Station de Radioastronomie de Nançay, Comptes Rendus, 249, 634

Lilley, A.E. and McClain, E.F. 1956, The Hydrogen-Line Red Shift of Radio Source Cygnus A, ApJ, 123, 172

Lovell, A.C.B. 1984a, Impact of World War II on Radio Astronomy. In Serendipitous Discoveries in Radio Astronomy, ed. K.I. Kellermann and B. Sheets (Green Bank: NRAO/AUI), 89

Lovell, A.C.B. 1984b, The Origins and Early History of Jodrell Bank. In The Early Years of Radio Astronomy, ed. W.T. Sullivan III (Cambridge: CUP)

Lovell, A.C.B. 1990, Astronomer by Chance (New York: Basic Books Inc.)

Mayer, C.H., McCullough, T.P., and Sloanaker, R.M. 1957, Evidence for Polarized Radio Radiation from the Crab Nebula, $A p J, 126,468$

Mayer, C.H., McCullough, T.P., and Sloanaker, R.M. 1958a, Observations of Venus at 3.15-CM Wave Length, ApJ, 127, 1

Mayer, C.H., McCullough, T.P., and Sloanaker, R.M. 1958b, Observation of Mars and Jupiter at a Wave Length of $3.15 \mathrm{~cm}, A p J, 127,11$

McClain, E.F. 1958, The Naval Research Laboratory's 84-foot Radio Telescope, SerT, $17(12), 608$

McCready, L.L. et al. 1947, Solar Radiation at Radio Frequencies and its Relation to Sunspots, Proc. Roy. Soc. A, 190, 357

Mills, B.Y. 1984, Radio Sources and the Log N - Log S Controversy. In The Early Years of Radio Astronomy, ed. W.T. Sullivan III (Cambridge: CUP)

Mills, B.Y. 2006, An Engineer Becomes an Astronomer, ARAA, 44, 1

Mills, B.Y. and Little, A.G. 1953, A High-Resolution Aerial System of a New Type, Austr. J. Phys., 6, 272

Mills, B.Y. and Slee, O.B. 1957, A Preliminary Survey of Radio Sources in a Limited Region of the Sky at a Wavelength of $3.5 \mathrm{~m}$, Austr. J. Phys., 10, 162 
Mills, B.Y., Slee, O.B., and Hill, E.R. 1960, A Catalogue of Radio Sources between Declinations $-20^{\circ}$ and $-50^{\circ}$, Austr. J. Phys., 13, 676

Mills, B.Y. and Thomas, A.B. 1951, Observations of the Source of Radio-Frequency Radiation in the Constellation of Cygnus, Austr. J. Sci. Res. A, 4, 158

Muller, C.A. and Oort, J.H. 1951, The Interstellar Hydrogen Line at 1,420 Mc./sec and an Estimate of Galactic Rotation, Nature, 168, 356

Needell, A.A. 2000, Science, Cold War and the American State, Lloyd V. Berkner and the Balance of Professional Ideals (Amsterdam: Harwood Academic)

Orchiston, W. 2005, Dr. Elizabeth Alexander: First Female Radio Astronomer. In The New Astronomy, ed. W. Orchiston (Dordrecht: Springer), 71

Orchiston, W. et al. 2007, Highlighting the History of French Radio Astronomy, $J A H H, 10,221$

Pawsey, J.L. 1946, Observations of Million Degree Thermal Radiation from the Sun at a Wavelength of 1.5 Metres, Nature, 158, 633

Penzias, A. and Wilson, R.W. 1965, A Measurement of Excess Antenna Temperature at $4080 \mathrm{Mc} / \mathrm{s}$, ApJ, 142, 419

Perlmutter, S. et al. 1999, Measurements of Omega and Lambda from 42 HighRedshift Supernovae, ApJ, 517, 565

Radhakrishnan, V. 2006, Olof Rydbeck and Early Swedish Radio Astronomy, $J A H H, 9,139$

Reber, G. 1944, Cosmic Static, ApJ, 100, 279

Reber, G. 1946, Solar Radiation in 489 Mc/sec, Nature, 158, 945

Reber, G. 1955, Tropospheric Refraction near Hawaii, IRE Trans. Ant. Prop., AP-3, 143

Reber, G. 1958, Early Radio Astronomy at Wheaton, Illinois, Proc. IRE, 46, 15

Reber, G. 1959, Radio Interferometry at Three Kilometers Altitude Above the Pacific Ocean, JGR, 64, 287

Reber, G. and Greenstein, J.L. 1947, Radio Frequency Investigations of Astronomical Interest, Observatory, 67, 15

Riess, A.G. et al. 1998, Observational Evidence from Supernovae for an Accelerating Universe and a Cosmological Constant, $A J, 116,1009$

Rigden, J.S. 1987, Rabi Scientist and Citizen (New York: Basic Books)

Robertson, P. 1992, Beyond Southern Skies - Radio Astronomy and the Parkes Telescope (Cambridge: CUP)

Robertson, P. 2017, Radio Astronomer-John Bolton and a New Window on the Universe (Sydney: NewSouth Publishing)

Rogers, A.E.E. 2007, Observations of the $327 \mathrm{MHz}$ Deuterium Hyperfine Transition, $A J, 133,1625$

Ryle, M. 1955, Radio Stars and Their Cosmological Significance, Observatory, 75, 137

Ryle, M. 1958, Bakerian Lecture. The Nature of the Cosmic Radio Sources, Proc. Roy. Soc., 248, 289

Ryle, M. 1968, Counts of Radio Sources, ARAA, 6, 249

Ryle, M. and Clarke, R.W. 1961, An Examination of the Steady-State Model in the Light of Some Recent Observations of Radio Sources, MNRAS, 122, 349

Ryle, M. and Scheuer, P.A.G. 1955, The Spatial Distribution and Nature of Radio Stars, Proc. Roy. Soc., 230, 448

Ryle, M., Smith, F.G., and Elsmore, B. 1950, A Preliminary Survey of the Radio Stars in the Northern Hemisphere, MNRAS, 110, 508

Salomonovich, A.E. 1984, The First Steps of Soviet Radio Astronomy. In The Early Years of Radio Astronomy, ed. W.T. Sullivan III (Cambridge: CUP), 269 
Scheuer, P.A.G. 1957, A Statistical Method for Analyzing Observations of Faint Radio Stars, Proc. Camb. Phil. Soc., 53, 764

Schott, E. 1947, 175 MHz Solar Radiation, Phys. Blatter, 3 (5), 159

Seeger, C.L. and Williamson, R.E. 1951, The Pole of the Galaxy as Determined from Measurements at $205 \mathrm{Mc} / \mathrm{sec}, A p J, 113,21$

Shakeshaft, J.R. et al. 1955, A Survey of Radio Sources Between Declinations $-38^{\circ}$ and $+83^{\circ}$, MNRAS, 67, 106

Shklovsky, I.S. 1949, Monochromatic Radio Emission from the Galaxy and the Possibility of Its Observation, Astr. Zh., 26, 10. English translation in Sullivan, W.T. III 1982, Classics in Radio Astronomy (Cambridge: CUP), 318

Shklovsky, I.S. 1960, Cosmic Radio Waves (Cambridge: Harvard Univ. Press)

Shklovsky, I.S., 1982, On the History of the Development of Radio Astronomy in the USSR, News on Life, Science, and Technology. No. 11 (Moscow: Izd. Znanie), 82 (in Russian)

Southworth, G. 1945, Microwave Radiation from the Sun, J. Franklin Inst. 239, 285

Southworth, G.C. 1956, Early History of Radio Astronomy, Sci. Monthly, 82 (2), 55

Stahr, M. 1949, The Variation of Solar Radiation at 205 Megacycles, AJ, 54, 195

Sullivan, W.T. III 1982, Classics in Radio Astronomy (Cambridge: CUP)

Sullivan, W.T. III ed. 1984, The Early Years of Radio Astronomy (Cambridge: CUP)

Sullivan, W.T. III 2009, Cosmic Noise: A History of Early Radio Astronomy (Cambridge: CUP)

Sullivan, W., Goss, W.M., and Raj, A. 2019, A "Radio Sundial" for the Jansky Very Large Array in New Mexico, Compendium, 23 (3) https://science.nrao.edu/ about/publications/sundial

Tanaka, H. 1984, Development of Solar Radio Astronomy in Japan up until 1960. In The Early Years of Radio Astronomy, ed. W.T. Sullivan III (Cambridge: CUP), 335

Townes, C.H. 1957, Microwave and Radio-Frequency Resonance Lines of Interest to Radio Astronomy. In IAU Symposium No.4, Radio Astronomy, ed. H.C. van de Hulst (Cambridge: CUP)

van de Hulst, H.C. 1945, Origin of Radio Waves from Space, Nederlandsch Tijdschrift voor Natuurkunde, 11, 210. English translation in Sullivan, W.T. III 1982, Classics in Radio Astronomy (Cambridge: CUP), 302

Wang, S. 2009, Personal Recollections of W.N. Christiansen and the Early Days of Chinese Radio Astronomy, JAHH, 12, 33

Wild, J.P. 1952, The Radio-Frequency Line Spectrum of Atomic Hydrogen and Its Applications in Astronomy, ApJ, 115, 206

Wild, J.P. 1967, The Radioheliograph and the Radio Astronomy Programme of the Culgoora Observatory, Proc. Astron. Soc. Austr., 1, 38

\section{FURTHER READING}

Allison, D.K. 1981, New Eye for the Navy: The Origin of Radar at the Naval Research Laboratory (Washington, NRL) https://apps.dtic.mil/dtic/tr/fulltext/u2/ al10586.pdf

Bolton, J.G. 1960, Radio Telescopes. In Telescopes, Stars and Stellar Systems, 1, ed. G.P. Kuiper and B.M. Middlehurst (Chicago: University of Chicago Press), 176

Bok, B.J. 1956, The George R. Agassiz Radio Telescope of the Harvard Observatory, Nature, 178, 232 
Bok, B.J., Ewen, H.I., and Heeschen, D.S. 1957, The George R. Agassiz Radio Telescope of Harvard Observatory, AJ, 62, 8

Conklin, N.D. 2006, Two Paths to Heaven's Gate (Charlottesville: NRAO)

Heeschen, D.S. 1956, Harvard's New Radio Telescope, SerT, 15, 388

Kellermann, K.I. 1972, Radio Galaxies, Quasars, and Cosmology, AJ, 77, 531

Kraus, J.D. 1988, Grote Reber, Founder of Radio Astronomy, JRASC, 82, 107

Leverington, D. 2017, Observatories and Telescopes of Modern Times (Cambridge: CUP)

Levy, D.H. 1993, The Man Who Sold the Milky Way (Tucson: University of Arizona Press)

Orchiston, W. and Slee, O.B. 2005, The Radiophysics Field Stations, and the Early Development of Radio Astronomy. In The New Astronomy, ed. W. Orchiston (Dordrecht: Springer), 119

Scheuer, P.A.G. 1968, Radio Astronomy and Cosmology. In Stars and Stellar Systems, 9, ed. A.R. Sandage et al. (Chicago: University of Chicago Press), 725

Scheuer, P.A.G. 1990, Radio Source Counts. In Modern Cosmology in Retrospect, ed. B. Bertotti et al. (Cambridge: CUP), 331

Strom, R. 2005, Radio Astronomy in Holland before 1960. In The New Astronomy, ed. W. Orchiston (Dordrecht: Springer), 93

Sullivan, W.T. III 1990, The Entry of Radio Astronomy into Cosmology: Radio Stars and Martin Ryle's 2C Survey. In Modern Cosmology in Retrospect, ed. B. Bertotti et al. (Cambridge: CUP), 309

Van Worden, H. and Strom, R. 2006, The Beginnings of Radio Astronomy in the Netherlands, $J A H H, 9,3$

Weaver, H.F. 2006, Early Days of the Radio Astronomy Laboratory and the Hat Creek Radio Observatory. In ASPC 356, Revealing the Molecular Universe: One Antenna is not Enough, ed. D.C. Backer et al. (San Francisco: ASP), 75

Open Access This chapter is licensed under the terms of the Creative Commons Attribution 4.0 International License (http://creativecommons.org/licenses/ by/4.0/), which permits use, sharing, adaptation, distribution and reproduction in any medium or format, as long as you give appropriate credit to the original author(s) and the source, provide a link to the Creative Commons licence and indicate if changes were made.

The images or other third party material in this chapter are included in the chapter's Creative Commons licence, unless indicated otherwise in a credit line to the material. If material is not included in the chapter's Creative Commons licence and your intended use is not permitted by statutory regulation or exceeds the permitted use, you will need to obtain permission directly from the copyright holder. 